\title{
Auroral current systems in Saturn's magnetosphere: comparison of theoretical models with Cassini and HST observations
}

\author{
S. W. H. Cowley ${ }^{1}$, C. S. Arridge ${ }^{2,3}$, E. J. Bunce ${ }^{1}$, J. T. Clarke ${ }^{4}$, A. J. Coates $^{2,3}$, M. K. Dougherty ${ }^{5}$, J.-C. Gérard ${ }^{6}$, \\ D. Grodent ${ }^{6}$, J. D. Nichols ${ }^{4}$, and D. L. Talboys ${ }^{1}$ \\ ${ }^{1}$ Department of Physics \& Astronomy, University of Leicester, Leicester LE1 7RH, UK \\ ${ }^{2}$ Mullard Space Science Laboratory, University College London, Dorking RH5 6NT, UK \\ ${ }^{3}$ Centre for Planetary Sciences, University College London, Gower Street, London WC1E 6BT, UK \\ ${ }^{4}$ Center for Space Physics, Boston University, Boston, MA 02215, USA \\ ${ }^{5}$ Blackett Laboratory, Imperial College London, London SW7 2AZ, UK \\ ${ }^{6}$ Laboratoire de Physique Atmosphérique et Planétaire, Université de Liège, Liège 4000, Belgium
}

Received: 29 April 2008 - Accepted: 23 July 2008 - Published: 8 September 2008

\begin{abstract}
The first simultaneous observations of fields and plasmas in Saturn's high-latitude magnetosphere and UV images of the conjugate auroral oval were obtained by the Cassini spacecraft and the Hubble Space Telescope (HST) in January 2007. These data have shown that the southern auroral oval near noon maps to the dayside cusp boundary between open and closed field lines, associated with a major layer of upward-directed field-aligned current (Bunce et al., 2008). The results thus support earlier theoretical discussion and quantitative modelling of magnetosphereionosphere coupling at Saturn (Cowley et al., 2004), that suggests the oval is produced by electron acceleration in the field-aligned current layer required by rotational flow shear between strongly sub-corotating flow on open field lines and near-corotating flow on closed field lines. Here we quantitatively compare these modelling results (the "CBO" model) with the Cassini-HST data set. The comparison shows good qualitative agreement between model and data, the principal difference being that the model currents are too small by factors of about five, as determined from the magnetic perturbations observed by Cassini. This is suggested to be principally indicative of a more highly conducting summer southern ionosphere than was assumed in the CBO model. A revised model is therefore proposed in which the heightintegrated ionospheric Pedersen conductivity is increased by a factor of four from 1 to 4 mho, together with more minor adjustments to the co-latitude of the boundary, the flow shear across it, the width of the current layer, and the properties of the source electrons. It is shown that the revised model agrees well with the combined Cassini-HST data, re-
\end{abstract}

Correspondence to: S. W. H. Cowley

(swhc1@ion.le.ac.uk) quiring downward acceleration of outer magnetosphere electrons through a $\sim 10 \mathrm{kV}$ potential in the current layer at the open-closed field line boundary to produce an auroral oval of $\sim 1^{\circ}$ width with $\mathrm{UV}$ emission intensities of a few tens of $\mathrm{kR}$.

Keywords. Magnetospheric physics (Auroral phenomena; Magnetosphere-ionosphere interactions; Planetary magnetospheres)

\section{Introduction}

Observations of planetary auroras provide a means of remotely sensing global magnetospheric dynamics, projected along field lines into the polar upper atmosphere. While "diffuse" auroral emissions are formed by precipitation from hot plasma populations produced inside planetary magnetospheres, bright structured "discrete" auroras are related to the field-aligned current systems that couple momentum between the ionosphere and magnetosphere, specifically regions where current is directed upward from the ionosphere resulting in downward acceleration of hot magnetospheric electrons. It is generally understood that for the giant planets the principal momentum exchange is from planetary rotation to magnetospheric plasma, and that for the planetary field polarities present at both Jupiter and Saturn, upward currents flow where the plasma angular velocity decreases with increasing latitude. At such locations a bright "auroral oval" may form if the downward acceleration of magnetospheric electrons required by the density of the upwarddirected field-aligned current produces precipitating electron energy fluxes of sufficient intensity.

Published by Copernicus Publications on behalf of the European Geosciences Union. 
Within this general scenario, two basic processes have been discussed that may lead to a fall in plasma angular velocity with latitude, and the consequent formation of a ring of upward-directed field-aligned current. The first is plasma production, pick-up, and radial transport from internal gas sources such as planetary moons and rings, leading to sub-corotation of the magnetospheric plasma on closed magnetic field lines (e.g. Hill, 1979; Vasyliunas, 1983; Saur et al., 2004). This is the process believed to produce the "main oval" at Jupiter (Cowley and Bunce, 2001; Hill, 2001; Southwood and Kivelson, 2001), mapping magnetically into the middle magnetosphere (Clarke et al., 1998; Prangé et al., 1998). However, modelling of the same current system at Saturn based on Voyager plasma angular velocity measurements led Cowley and Bunce (2003) to conclude that the field-aligned currents are too weak in this case to result in significant electron acceleration and auroral emission, and also occur at too low latitude to account for the observed auroral oval. The second possibility is the flow shear expected to occur at the boundary between closed field lines that moderately sub-corotate, and open field lines in the polar region that strongly sub-corotate according to both theory and observation (Isbell et al., 1984; Stallard et al., 2003, 2004). Cowley et al. (2004a) thus suggested that Saturn's main oval maps to this upward current layer at the boundary between open and closed field lines, a suggestion shown in subsequent modelling to be plausible in terms of accelerated electron and auroral parameters (Cowley et al., 2004b; Jackman and Cowley, 2006). Similar processes may also occur at Jupiter, forming a component of the auroras lying poleward of the main oval (Cowley et al., 2005).

In addition to these scenarios that are associated with large-scale field-aligned currents and magnetosphereionosphere momentum coupling, Sittler et al. (2006) have also proposed that Saturn's auroral oval is produced by plasma heating and acceleration initiated by the interchange instability at the outer edge of Saturn's plasma sheet. In this case the oval will map magnetically to closed field lines within the outer part of the magnetosphere, which Sittler et al. (2006) suggest to lie typically at $\sim 15 R_{S}$ in the equatorial plane (Saturn's radius, $R_{S}$, is equal to $60268 \mathrm{~km}$.). These field lines thus usually lie several Saturn radii inside the dayside magnetopause, the latter boundary being typically located at $\sim 20-25 R_{S}$ in the sub-solar region, depending on the dynamic pressure of the solar wind (Arridge et al., 2006).

Badman et al. (2006) have determined the average position of Saturn's main oval auroras in the Southern Hemisphere from sets of UV images obtained by the Hubble Space Telescope (HST) (e.g. Gérard et al., 2004; Clarke et al., 2005; Grodent et al., 2005). Mapping these locations along model field lines shows that the emissions relate to the outer magnetosphere and magnetopause vicinity beyond the corotation breakdown region in the middle magnetosphere ring current (Badman et al., 2006; Bunce et al., 2008a). These results are thus in agreement with the initial modelling results of
Cowley and Bunce (2003), but do not clearly discriminate the locations proposed by Cowley et al. (2004a, b) and Sittler et al. (2006). Recently, however, more direct investigation of the origins of Saturn's auroras has become possible through HST imaging coordinated with in situ observations by Cassini in the high-latitude magnetosphere (Clarke et al., 2008 ${ }^{1}$ ), when the spacecraft crossed field lines mapping to the auroral oval and polar cap. In the first such study Bunce et al. (2008b) have shown using Cassini field and plasma data that the UV oval observed near noon indeed maps to the boundary between open and closed field lines, where a major layer of upward-directed field-aligned current flows out of the ionosphere, thus supporting the discussion of Cowley et al. (2004a) and the model studies of Cowley et al. (2004b).

The purpose of this paper is to present a comparison of the unique combined Cassini-HST observations discussed by Bunce et al. (2008b) with the model results of Cowley et al. (2004b). It is shown that while the model is in good qualitative accord with the data, some parameters require adjustment to provide detailed quantitative agreement.

\section{Overview of Cassini and HST observations}

Following orbit insertion in mid-2004, the early phase of the Cassini mission was confined to the near-equatorial regions of Saturn's magnetosphere. However, beginning in mid-2006 the orbit was progressively tilted out of the equatorial plane, allowing exploration of higher latitudes. In January 2007 a sequence of daily observations of Saturn's UV auroras was undertaken using the HST, coordinated with high-latitude Cassini observations during Rev 37 of the planet. The subset of these data discussed by Bunce et al. (2008b) that forms the basis of the modelling comparison presented here is summarised in Figs. 1 and 2 for HST and Cassini data, respectively, and will be overviewed in this section prior to discussion of the theoretical modelling in Sects. 3-5.

The auroral images in Fig. 1 were obtained using the solarblind channel of the Advanced Camera for Surveys (ACS) on consecutive HST "visits" on 16 and 17 January 2004, and show UV emissions in the southern polar region colourcoded according to the intensity scale on the right. See Clarke et al. (2008) $)^{1}$ for an overview of the overall HST campaign. The images are projected onto a latitude-local time grid as though viewed through the planet looking from the north, with noon at the bottom and dawn to the left. We note that Saturn's southern pole was tilted toward the Earth (and HST) by $\sim 13^{\circ}$ during this interval, giving a reasonable view

\footnotetext{
${ }^{1}$ Clarke, J. T., Nichols, J. D., Gérard, J.-C., Grodent, D., Hansen, K. C., Kurth, W. R., Gladstone, G. R., Duval, J., Wannawichian, S., Bunce, E. J., Cowley, S. W. H., Crary, F. J., Dougherty, M. K., Lamy, L., Mitchell, D., Pryor, W., Retherford, K., Stallard, T. S., and Zieger, B.: The response of Jupiter's and Saturn's auroral activity to the solar wind, J. Geophys. Res., in review, 2008.
} 
of the southern emissions, but an inadequate view of auroras in the north. Even so, the images in Fig. 1 are truncated on the nightside due to the uncertainty in the polar projections near the planet's limb. Dotted circles in the figure are plotted at $5^{\circ}$ intervals of co-latitude from the southern pole. Each image shows the sum of five individual exposures, obtained for image A during 05:31-05:41 UT "Saturn time" (HST time minus 69 min light travel time from Saturn) on 16 January, and for image B during 03:21-03:30 UT on 17 January. It can be seen that a relatively narrow and well-defined auroral oval was present from dawn to noon in both images, with broader and somewhat more scattered emissions being present in the post-noon and dusk sector. It can also be seen that the emissions contracted poleward somewhat during the $\sim 22 \mathrm{~h}$ interval between the two images.

The superposed white lines in the panels of Fig. 1 show the trajectory of the Cassini spacecraft mapped along magnetic field lines into the southern ionosphere. The magnetic model employed consists of the sum of the "Cassini" internal field model (Dougherty et al., 2005), and a typical ring current field determined from Cassini data by Bunce et al. (2007) (specifically for a typical assumed subsolar magnetopause position of $21 R_{S}$, though other choices make only marginal differences). Dots are shown every six hours along the trajectory, with the start of days indicated by the "day of year" (DOY) markers. During the relevant interval spanning the images on days 16-17 the spacecraft was located at nearconstant radial distances of $\sim 13 R_{S}$ in the Southern Hemisphere mapping to high latitudes in the conjugate southern ionosphere, and moving from dawn to the post-noon sector (see further in Fig. 2). The mapped location of the spacecraft at the centre time of each image is shown by the red dots plotted on the trajectory. It can be seen from these locations that at the time of image A the spacecraft footprint was located in the "polar cap" well poleward of the auroral emissions, at $\sim 09: 00 \mathrm{LT}$ and $\sim 8.5^{\circ}$ co-latitude with respect to the southern pole. However, by the time of image B the footprint had moved just equatorward of the emissions, then being located at $\sim 13: 00 \mathrm{LT}$ and $\sim 14.5^{\circ}$ co-latitude. These results thus show that in the interval between images A and B, Cassini traversed magnetospheric field lines mapping to Saturn's southern auroral oval, from poleward to equatorward in the noon sector.

In Fig. 2 we show Cassini plasma electron and magnetic field data spanning the times of the two images in Fig. 1, specifically for the $42 \mathrm{~h}$ interval from 18:00 UT on 15 January to 12:00 UT on 17 January 2007. Spacecraft position data are shown at the foot of the figure. From top to bottom these give the spacecraft local time (LT in decimal hours), the co-latitude relative to the northern spin and magnetic axis (Co-Lat in deg), the radial distance from the planet's centre ( $R$ in $R_{S}$ ), and the magnetically mapped co-latitude of the spacecraft in the southern ionosphere as in Fig. $1\left(\theta_{i S H}\right.$ in deg). The top panel of Fig. 2 then shows an electron spectrogram from $0.6 \mathrm{eV}$ to $26 \mathrm{keV}$ obtained by the CAPS-ELS elec- (a) 05:31-05:41 UT 16 Jan 2007

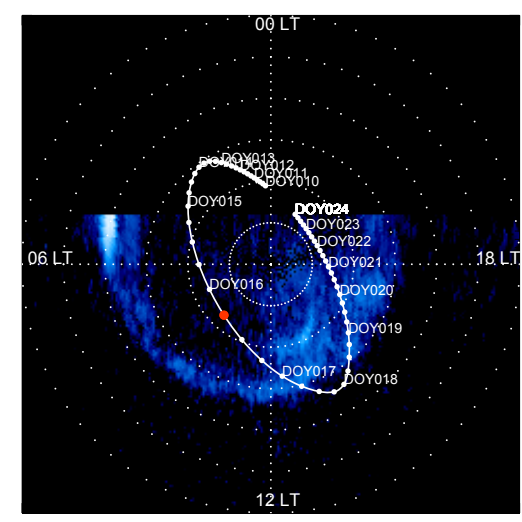

(b) 03:21-03:30 UT 17 Jan 2007

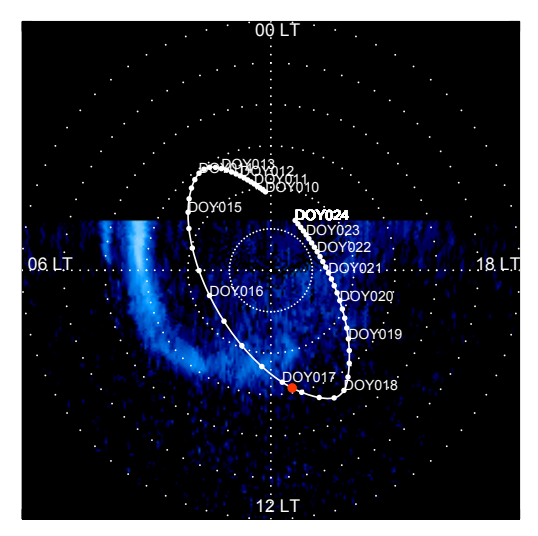

Fig. 1. Two HST/ACS UV images of Saturn's southern auroras obtained on consecutive "visits" in January 2007. The emissions are colour-coded according to the intensity scale on the right, and are projected onto a latitude-local time grid as though viewed through the planet looking from the north, with noon at the bottom and dawn to the left. Dotted circles are plotted at $5^{\circ}$ co-latitude intervals from the southern pole. The images are truncated on the nightside due to the uncertainty in the polar projections near the planet's limb. Image A (upper panel) was obtained in the interval 05:31-05:41 UT "Saturn time" (HST time minus 69 min light travel time from Saturn) on 16 January, while image B (lower panel) was obtained in the interval 03:21-03:30 UT "Saturn time" on 17 January. The superposed solid lines show the ionospheric footprint of Cassini mapped magnetically to the ionosphere using the internal planetary field combined with a model ring current field as described in the text. White dots are plotted on the trajectory every six hours, with the start of each day indicated by the "day of year" (DOY) number. The mapped spacecraft position at the time at which each image was obtained is indicated by the red dot in each panel (after Bunce et al., 2008b).

tron spectrometer (Young et al., 2004), colour-coded according to the scale on the right. We note that the counts at lowest energies are mainly spacecraft photoelectrons (at energies $\sim 30 \mathrm{eV}$ and below during the early part of the interval, but 


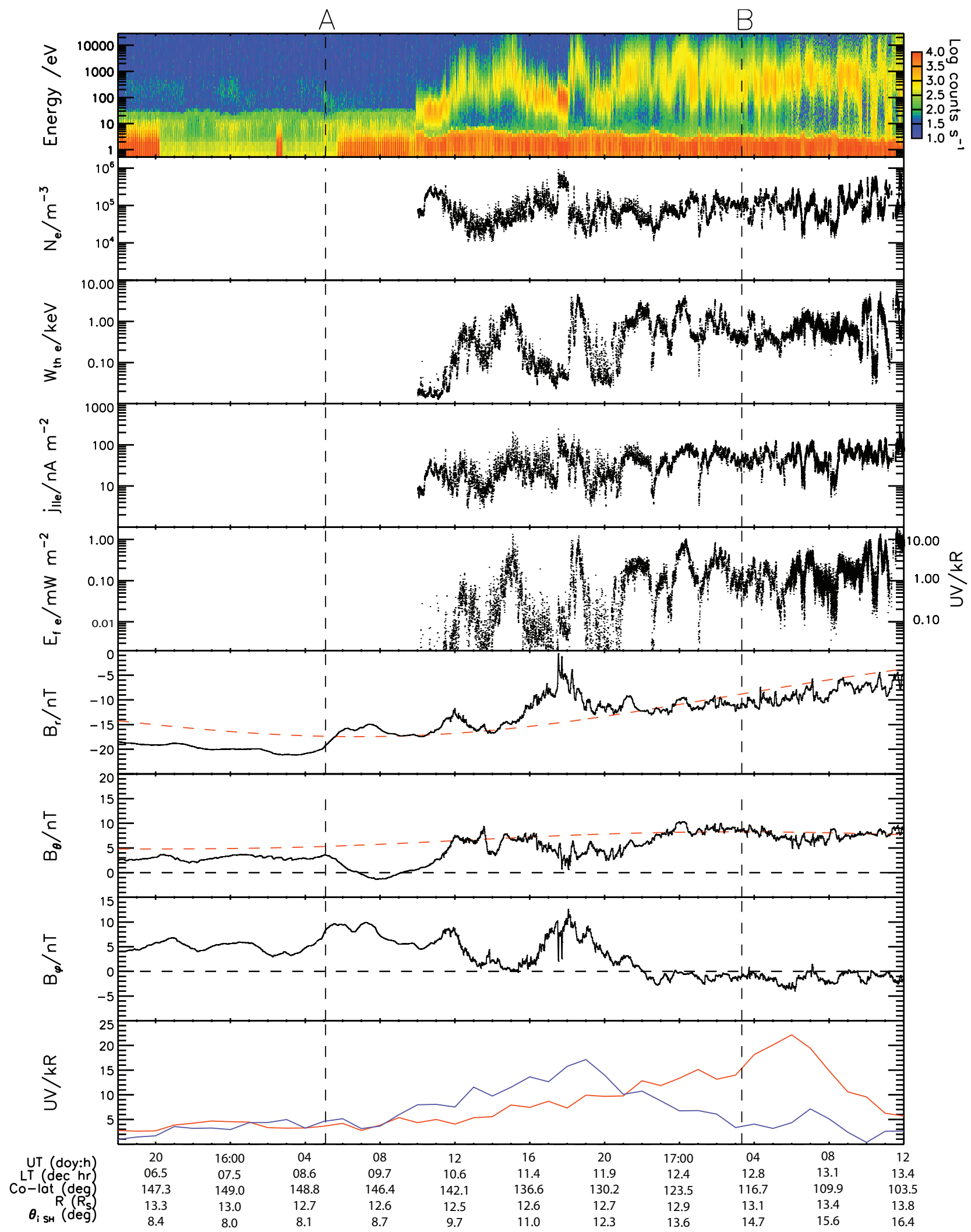

Fig. 2. Caption on next page. 
Fig. 2. Overview of Cassini plasma electron and magnetic field observations obtained during the $42 \mathrm{~h}$ interval on Rev $37 \mathrm{between} 18: 00 \mathrm{UT}$ on 15 January and 12:00 UT on 17 January 2007, spanning the times of images A and B shown in Fig. 1. The centre times at which these images were obtained are marked by the vertical dashed lines marked A and B at the top of the figure. The top five panels show electron data obtained by the Cassini CAPS-ELS instrument, specifically an electron spectrogram from $\sim 0.6 \mathrm{eV}$ to $\sim 26 \mathrm{keV}$ colour-coded according to the scale on the right (the counts at low energies are mainly spacecraft photoelectrons), followed by plots of bulk parameters obtained by numerical integration over the electron distribution (excluding photoelectrons and background contamination) assuming the distribution is isotropic. Bulk parameter values are not shown before $\sim 10: 00$ UT on 16 January due to low electron fluxes resulting in low measurement signal-to-noise. The bulk parameters shown are the electron density $N_{e}$, the thermal energy $W_{t h e}$, the current density of electrons moving in one direction along the field lines $j_{\| e}$ (the field-aligned number flux of these electrons times the electron charge), and the corresponding field-aligned energy flux of these electrons $E_{f e}$ (see text for further explanation of the last two parameters). The right-hand scale on the energy flux panel shows the corresponding UV auroral emission expected if these electrons precipitate into the atmosphere unmodified by field-aligned acceleration. The sixth to eighth panels show the three components of the magnetic field in spherical polar coordinates referenced to the planet's spin and magnetic axis. The red dashed lines in the $B_{r}$ and $B_{\theta}$ panels show the "Cassini" internal planetary field of Dougherty et al. (2005). No such line is shown in the $B_{\varphi}$ panel, since the model planetary field is exactly axi-symmetric with zero azimuthal component. The bottom panel shows the UV auroral intensity at the ionospheric footprint of the spacecraft in the Southern Hemisphere obtained from the two HST images shown in Fig. 1, mapped magnetically as for the spacecraft footprint in the latter figure. The red line corresponds to image A and the blue to image B. Spacecraft position data are given at the foot of the figure, specifically the local time (LT in decimal hours), the co-latitude with respect to the northern spin and magnetic axes (Co-Lat in degrees), the radial distance of the spacecraft ( $R$ in $R_{S}$ ), and the magnetically mapped co-latitude in the southern ionosphere as employed in Fig. 1 ( $\theta_{i} S H$ in degrees) (adapted from Bunce et al., 2008b).

at less than $\sim 10 \mathrm{eV}$ and below after $\sim 10$ :00 UT on 16 January). The four panels beneath this then show electron bulk parameters obtained by numerical integration over the observed distributions (Lewis et al., 2008), where we have assumed that the observed populations are near-isotropic, and contributions from photoelectrons and background contamination have been removed. Values are not displayed prior to $\sim$ 10:00 UT on 16 January due to low electron fluxes at the spacecraft, such that it is impossible to derive reliable moments with good time resolution. From top to bottom we show the electron density $N_{e}$, the thermal energy $W_{\text {th } e}$, the field-aligned current density associated with electrons travelling in one direction only along the magnetic field lines $j_{\| e}$, and the corresponding field-aligned electron energy flux $E_{f e}$. The last two quantities are specifically the velocityspace integrals of $e v_{\|} f_{e}$ and $\left(m_{e} v^{2} / 2\right) v_{\|} f_{e}$ over one hemisphere only of the assumed near-isotropic populations, where $e$ and $m_{e}$ are the electron charge and mass, $v$ and $v_{\|}$are the electron speed and its field-aligned component, and $f_{e}$ is the electron distribution function. If the electrons are nearisotropic, as assumed, then the distribution function will be nearly independent of position along field lines down to the ionosphere, assuming no acceleration processes act to modify the precipitating electron distribution at lower altitudes. In this case, the quantities computed correspond to the current and energy flux delivered by the precipitating electrons to the ionosphere as discussed further in Sect. 3 below. The scale on the right hand side of the energy flux panel then estimates the resulting UV auroral emission, on the basis that $1 \mathrm{~mW} \mathrm{~m}^{-2}$ produces $\sim 10 \mathrm{kR}$ (e.g. Rego et al., 1994). In the magnetosphere itself, of course, the total electron current and net energy flux will be close to zero for a near-isotropic population, the values shown in the figure being near-exactly can- celled by electrons moving in the opposite direction along the field. Beneath the CAPS/ELS data we also show magnetic data obtained by the Cassini magnetic field investigation (Dougherty et al., 2004), specifically the three components of the magnetic field in spherical polar coordinates referenced to the planet's spin and magnetic axis, where for comparison the red dashed lines show the "Cassini" model planetary field. No red line is shown in the $B_{\varphi}$ panel, since the planetary field is closely axi-symmetric and has no measurable azimuthal component. In the bottom panel of Fig. 2 we also show the UV intensity at the spacecraft footprint in the southern ionosphere obtained from the two images shown in Fig. 1, where the red line corresponds to image $\mathrm{A}$ and the blue to image B. These plots thus indicate the UV intensity at the spacecraft footprint versus time if the emissions remained fixed at those observed in images A or B. The emission peaks corresponding to the traversal of the spacecraft footprint across the auroral ovals in the two images are evident.

The centre times of the two HST images are marked by the vertical dashed lines labelled A and B at the top of the Fig. 2, the interval between them thus spanning the crossing of the auroral oval according to the above discussion. As noted above, at the time of image A the spacecraft footprint in the southern ionosphere was located well inside the main oval in the mid-morning sector, as also indicated in Fig. 2 by the low UV intensities at the spacecraft footprint at that time shown in the bottom panel (red trace). The Cassini data at this time show a lack of measurable hot electron fluxes together with correspondingly "quiet" magnetic field components, as had been the case for $\sim 40 \mathrm{~h}$ previously (see Bunce et al., 2008b). These conditions indicate that the spacecraft was then located on open field lines inside the auroral oval, mapping 
to Saturn's southern tail lobe. After this time, the spacecraft moved increasingly toward the equator in the noon and postnoon sector, and to larger ionospheric co-latitudes relative to the southern pole (Fig. 1). At $\sim 10: 00$ UT on 16 January, prior to the time of image $B$, intense fluxes of warm electrons and "disturbed" field components began to be detected, first a structured region of cool magnetosheath-like electrons and hot $\mathrm{keV}$ electrons, followed after $\sim 21: 00$ UT by continuous but variable hot electrons, indicative of hot trapped plasma in the dayside outer magnetosphere. As discussed by Bunce et al. (2008b), this field and plasma sequence indicates that during this interval the spacecraft crossed the open-closed field line boundary between the tail lobe and outer dayside magnetosphere, in the region of the dayside cusp in the immediate pre-noon sector. At the time of image B, Cassini was thus located on closed field lines in the outer dayside magnetosphere, with its footprint lying just equatorward of the oval according to image $\mathrm{B}$ and the blue trace in the bottom panel of Fig. 2. This location is not inconsistent with the presence of hot electrons at the spacecraft, however, since examination of the electron energy flux values in Fig. 2 shows that the in situ populations at this time were capable of producing only sub-kR UV emissions upon precipitation, much less than those of the main oval in Fig. 1.

The in situ data in Fig. 2 thus show that the traversal of the main oval field lines near noon between the times of images $\mathrm{A}$ and $\mathrm{B}$ is associated with the dayside cusp transition between open and closed field lines. However, it is also evident that the oval is not simply associated with precipitation of magnetospheric electrons from the boundary region unmodified into the atmosphere, since examination of the electron energy flux values observed between the times of images A and B in Fig. 2 again shows that they are sufficient to produce UV emissions typically from a few tenths to a few $\mathrm{kR}$ in intensity, much less than the peak oval emissions of $\sim 15-20 \mathrm{kR}$ observed along the spacecraft track (bottom panel of Fig. 2). The conclusion thus follows that the oval emissions must be due electrons from the observed source populations between the times of images A and B being accelerated along the field lines in the boundary region into the ionosphere at altitudes below that of the spacecraft (located at $\sim 13 R_{S}$ ). As argued by Bunce et al. (2008b), the origin and nature of the acceleration process is also clearly indicated in the magnetic data obtained between the times of images A and B in Fig. 2, specifically in the behaviour of the azimuthal field component $B_{\varphi}$ shown in panel eight. As indicated above, due to its close rotational symmetry about the spin axis the internal planetary field has no measurable azimuthal component, and neither does the near axi-symmetric ring current field. Nevertheless a strong positive $B_{\varphi}$ is observed throughout the region of open field lines poleward of the cusp, which, with significant temporal or spatial structure, drops across the structured cusp region containing cool magnetosheath-like electrons to small negative values in the dayside outer magnetosphere region containing the variable hot electron fluxes. As Bunce et al. (2008b) point out, and as will be discussed further below, this is the magnetic signature of a major layer of field-aligned current directed upward out of the southern ionosphere. The total current flowing in the layer is $\sim 4-5$ MA per radian of azimuth, and with an estimated layer width of $\sim 1.5^{\circ}-2^{\circ}$ in the ionosphere, the field-aligned current density just above the ionosphere is estimated to be $\sim 200-300 \mathrm{nA} \mathrm{m}^{-2}$. As can be seen in the fourth panel of Fig. 2, such current densities typically exceed those that can be provided to the ionosphere by the unaccelerated source populations by around an order of magnitude, thus implying that these electrons must be accelerated into the ionosphere to carry the observed current, thus producing the enhanced UV emissions of the noon oval. Indeed, Bunce et al. (2008b) show using the kinetic theory of Knight (1973) that the source electrons must be accelerated along the field through voltages of typically several $\mathrm{kV}$ to produce such current densities, thus amplifying the precipitating electron energy flux to values capable of producing the few tens of $k R$ emissions observed. With regard to the emissions observed along the spacecraft footprint shown in the bottom panel of Fig. 2, it can be seen that peak intensities are $\sim 15-20 \mathrm{kR}$ centred in a region of total half-power width $\sim 3^{\circ}$ co-latitude in the southern ionosphere for both images. However, it should be recognised that the emission distribution will inevitably have been somewhat spread in these images due both to the finite resolution of the instrument, and by projection effects. The instrument resolution contributes $\sim 1^{\circ}$ spread near the noon oval, while projection effects, particularly due to the finite height of the auroral curtain, likely contributes a further $\sim 1.5^{\circ}$. The overall co-latitude spread due to both these effects is thus likely to be $\sim 2^{\circ}$ in the vicinity of the noon oval. It is thus clear that the actual auroral distribution along the spacecraft track could have been significantly narrower than that indicated by the UV profiles in the bottom panel of Fig. 2.

It is evident from this description that the data in Fig. 2 are qualitatively in conformity with the theoretical scenario presented by Cowley et al. (2004a), in which Saturn's main oval maps to upward field-aligned currents flowing in the openclosed field line boundary region. However, a quantitative comparison is clearly desirable. In the following sections we thus compare these data with the axi-symmetric model of Cowley et al. (2004b), as seems appropriate to a first discussion.

\section{The CBO model}

We begin in this section by presenting an overview of the Saturn magnetosphere-ionosphere coupling model of Cowley et al. (2004b), which for brevity we term the "CBO" model. Full details are provided in that paper and by Cowley and Bunce (2003), such that only an outline will be given here. The nature of the calculation is essentially simple, based on 
an initial assumption of axi-symmetry of the magnetic field and the plasma flow. First, a model of the plasma angular velocity on magnetospheric magnetic field lines is constructed, based principally on Voyager plasma velocity data and theoretical considerations. This is then combined with model ionospheric parameters to compute the equatorward-directed horizontal ionospheric Pedersen current intensity versus colatitude. The divergence of this current then gives the fieldaligned current density just above the ionosphere required by current continuity, extending along field lines into the magnetosphere, from which the auroral acceleration parameters are calculated using Knight's (1973) kinetic theory and a model of the magnetospheric source electron parameters.

In somewhat more detail, the model plasma angular velocity is defined as a function of the magnetic flux function $F(r, \theta)$, related to the poloidal field components by $\boldsymbol{B}=(1 / r \sin \theta) \nabla F \times \hat{\boldsymbol{\varphi}}$, where we use spherical polar coordinates referenced to the planet's spin and magnetic axes. Flux function $F$ is constant on magnetic field lines, and thus so is the plasma angular velocity, as required for a steadystate axi-symmetric model. In the ionosphere, where the effect of external current systems can be ignored (e.g. tail, magnetopause, and ring current), the field is taken to consist of axially-aligned and azimuthally symmetric internal dipole, quadrupole, and octupole terms, with north-south symmetry being broken by the quadrupole. The flux function for the dipole component, for example, is given by $F_{\text {dip }}(r, \theta)=g_{1}^{0} R_{S}^{2} \sin ^{2} \theta\left(R_{S} / r\right)$. The magnetic coefficients used here are those of the "Cassini" model of Dougherty et al. (2005) (i.e. $g_{1}^{0}=21084 \mathrm{nT}$ with $R_{S}=60268 \mathrm{~km}$ for the dipole component, for example), as also employed in the field mapping in Figs. 1 and 2. The previous papers by Cowley and Bunce (2003) and Cowley et al. (2004b) employed the earlier SPV model of Davis and Smith (1990), but these models differ only at the $\sim 1 \%$ level, which therefore does not significantly affect the results presented. Defining the arbitrary constant in the flux function such that its value is zero on the polar axis and increases monotonically towards a maximum close to the equator, the specific function employed to model the plasma angular velocity $\omega$, normalised to Saturn's angular velocity $\Omega_{S}$, is

$$
\begin{aligned}
\left(\frac{\omega(F)}{\Omega_{S}}\right)= & \left(\frac{\omega}{\Omega_{S}}\right)_{O} \\
& +\left(\left(\frac{\omega}{\Omega_{S}}\right)_{O M}-\left(\frac{\omega}{\Omega_{S}}\right)_{O}\right)\left[\frac{1}{2}\left(1+\tanh \left(\frac{F-F_{O}}{\Delta F_{O}}\right)\right)\right] \\
& +\left(\left(\frac{\omega}{\Omega_{S}}\right)_{M M}-\left(\frac{\omega}{\Omega_{S}}\right)_{O M}\right) \frac{1}{\left(1+\left(F_{1} / F\right)^{n_{1}}\right)} \\
& +\left(1-\left(\frac{\omega}{\Omega_{S}}\right)_{M M}\right) \frac{1}{\left(1+\left(F_{2} / F\right)^{n_{2}}\right)}
\end{aligned}
$$

where we directly follow the functional forms employed by Cowley and Bunce (2003) and Cowley et al. (2004b). In this expression $\left(\omega / \Omega_{S}\right)_{O}=0.3$ is the normalised angular velocity on open field lines at highest latitudes (smallest $F$ ) as indicated by the theory of Isbell et al. (1984) and the results of Stallard et al. (2004). This then switches sharply to the outer magnetosphere value $\left(\omega / \Omega_{S}\right)_{O M}=0.8$ across the open-closed field line boundary at $F \approx F_{O}=1526 \mathrm{nT} R_{S}^{2}$, corresponding ionospheric co-latitudes of $\sim 12.9^{\circ}$ in the north and $\sim 14.2^{\circ}$ in the south (with respect to the southern pole). The angular velocity switch takes place on a co-latitude scale of $\sim 0.5^{\circ}$, governed by parameter $\Delta F_{O}=50 \mathrm{nT} R_{S}^{2}$. As indicated by Voyager velocity data (Richardson, 1986; Richardson and Sittler, 1990), the normalised plasma angular velocity then falls toward middle-magnetosphere values of $\left(\omega / \Omega_{S}\right)_{M M}=0.6$ near $F \approx F_{1}=2200 \mathrm{nT} R_{S}^{2}\left(\sim 15.6^{\circ}\right.$ and $\sim 17.3^{\circ}$ in the north and south, respectively), on a scale governed by exponent $n_{1}=50$. It then increases gradually once more towards unity and rigid corotation at lower latitudes near $F \approx F_{2}=3600 \mathrm{nT} R_{S}^{2}\left(\sim 20.4^{\circ}\right.$ and $\sim 22.5^{\circ}$ in the north and south), governed by exponent $n_{2}=8$. Translated into variations versus co-latitude $\theta_{i}$ in the ionosphere, the resulting angular velocity profile is shown in Fig. $3 \mathrm{a}$, where the dashed line corresponds to the Northern Hemisphere, and the solid line to the Southern Hemisphere (plotted with respect to the southern pole). Here, as in Cowley and Bunce (2003), the ionosphere has been taken to correspond to a layer $1000 \mathrm{~km}$ above the planetary 1 bar reference spheroid. The sudden increase in plasma angular velocity across the open-closed field line boundary is a principal feature of the model. The subsequent drop in angular velocity between the outer and middle magnetosphere maps to a radial distance of $\sim 15 R_{S}$ in the equatorial plane in typical models (Bunce et al., 2008a). It thus corresponds to the outer part of the ring current region where Sittler et al. (2006) place the auroral oval on closed field lines.

Employing this model, the horizontal Pedersen current per radian of azimuth (longitude) flowing equatorward in the ionosphere is given by

$I_{h P}^{\prime}=\frac{\Sigma_{P}^{*} \rho_{i}^{2} \Omega_{S} B_{i}}{\cos \alpha_{i}}\left(1-\left(\frac{\omega}{\Omega_{S}}\right)\right)$,

where $\Sigma_{P}^{*}$ is the effective height-integrated ionospheric Pedersen conductivity (taking account of possible neutral atmosphere slippage from rigid corotation due to ion-neutral drag), $\rho_{i}$ the perpendicular distance of the ionospheric layer from the spin and magnetic axes, $B_{i}$ the field strength in the Pedersen layer, and $\alpha_{i}$ the angle of the ionospheric magnetic field to the local vertical. The value of $\Sigma_{P}^{*}$ is not well known, such that for simplicity a constant value of 1 mho was employed in the CBO model. However, we use an updated value of $\Omega_{S} \approx 1.615 \times 10^{-4} \mathrm{rad} \mathrm{s}^{-1}$ corresponding to a planetary period of $10.81 \mathrm{~h}$ (e.g. Kurth et al., 2007), though this again differs by only $\sim 1 \%$ from the value employed previously. The Northern and Southern Hemisphere Pedersen current profiles corresponding to the angular velocity model given by Eq. (1) is shown in Fig. 3b, in the same format as Fig. 3a. It can be seen that the current increases monotonically with colatitude on open field lines to $\sim 1$ MA per radian of azimuth 

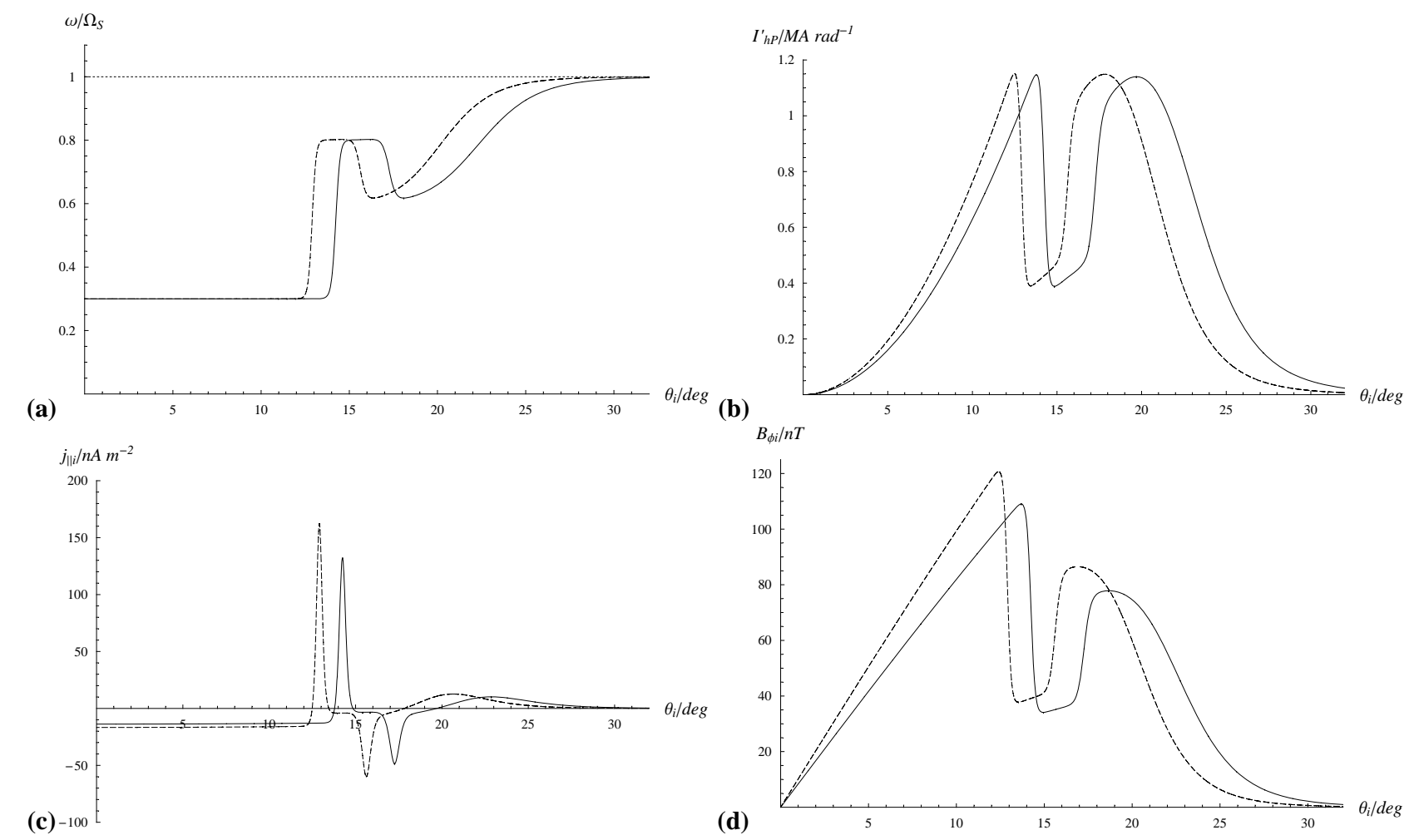

Fig. 3. Parameters of the CBO model of magnetosphere-ionosphere coupling at Saturn plotted versus co-latitude $\theta_{i}$ in the ionosphere for the Northern (dashed lines) and Southern Hemispheres (solid lines), the latter relative to the southern pole. Note that the plots shown here employ updated internal magnetic field and planetary angular velocity values compared with Cowley et al. (2004b), as described in the text, but that these result only in insignificant variations at the $\sim 1 \%$ level. The panels of the figure show (a) the plasma angular velocity $\omega$ normalised to the planet's angular velocity $\Omega_{S}$, given by Eq. (1), where the horizontal dotted line represents rigid corotation, (b) the equatorward-directed horizontal ionospheric Pedersen current per radian of azimuth $I_{h P}^{\prime}$, obtained from Eq. (2) with an effective ionospheric Pedersen conductivity of 1 mho, (c) the field-aligned current density just above the ionosphere $j_{\| i}$ required by the divergence of the horizontal Pedersen current, given by Eq. (3), where positive and negative values indicate upward and downward-directed currents, respectively, in both hemispheres, and (d) the azimuthal magnetic field $B_{\varphi i}$ immediately above the ionospheric Pedersen layer produced by the combined ionospheric current and field-aligned current system, given by Eq. (4), the value for the Northern Hemisphere being reversed in sign for convenience of plotting.

at the open-closed field line boundary, falls rapidly at the latter boundary where the plasma angular velocity increases, grows again to similar values across the outer and middle magnetosphere, and falls to small values as rigid corotation is approached at larger co-latitudes.

The model field-aligned current density just above the ionosphere then follows immediately from current continuity, given by

$j_{\| i}=-\frac{1}{\rho_{i} \cos \alpha_{i}} \frac{d I_{h P}^{\prime}}{d s_{i}}$,

where $d s_{i}$ is a meridional element of path length in the ionosphere from the pole toward the equator (see Cowley and Bunce, 2003, for details). The field-aligned current is thus directed downward into the ionosphere (negative values) where $I_{h P}^{\prime}$ rises with co-latitude from the respective pole, and upward out of the ionosphere (positive) where $I_{h P}^{\prime}$ falls. These currents for the CBO model are shown in
Fig. 3c, and correspondingly show weak $\left(\sim 15 \mathrm{nA} \mathrm{m}^{-2}\right)$ nearconstant downward currents throughout the region of open field lines, strong $\left(\sim 100 \mathrm{nA} \mathrm{m}^{-2}\right)$ upward currents in a narrow layer at the open-closed field line boundary, moderately strong $\left(\sim 50 \mathrm{nA} \mathrm{m}^{-2}\right)$ downward currents in the boundary between the outer and middle magnetosphere in the region where Sittler et al. (2006) place the auroral oval, and distributed weaker $\left(\sim 10 \mathrm{nA} \mathrm{m}^{-2}\right)$ upward currents in the middle magnetosphere. From the fall in the Pedersen current seen in second panel, the total current flowing up the field lines in the open-closed field line boundary in the model is $\sim 0.8 \mathrm{MA} \mathrm{rad}^{-1}$. A larger total current $\sim 1.2 \mathrm{MA} \mathrm{rad}^{-1}$ also flows up the field lines in the middle magnetosphere region, but because it is spread over a much larger area, the associated field-aligned current density is significantly less.

Field-aligned currents are very difficult to detect in situ in magnetospheric particle data (unlike their auroral effects below low-altitude acceleration regions), as will be illustrated 
Table 1. Properties of the magnetospheric source electron parameters employed in auroral calculations.

\begin{tabular}{lccc}
\hline Parameter & $\begin{array}{c}\text { Magnetosheath } \\
\text { CBO \& Revised }\end{array}$ & $\begin{array}{c}\text { Outer magnetosphere } \\
\text { CBO/Revised }\end{array}$ & $\begin{array}{c}\text { Middle magnetosphere } \\
\text { CBO \& Revised }\end{array}$ \\
\hline Electron density $N\left(\mathrm{~cm}^{-3}\right)$ & 0.2 & $0.01 / 0.04$ & 0.02 \\
Electron thermal energy $W_{t h}(\mathrm{keV})$ & 0.05 & $1.0 / 2.0$ & 0.15 \\
Unaccelerated current density $j_{\| i 0}\left(\mathrm{nA} \mathrm{m}^{-2}\right)$ & 37.9 & $8.48 / 48.0$ & 65.7 \\
Unaccelerated energy flux $E_{f 0}\left(\mathrm{~mW} \mathrm{~m}^{-2}\right)$ & 0.00379 & $0.0170 / 0.192$ & 0.0197 \\
\hline
\end{tabular}

in the results shown in Sect. 4 below. However, they do produce a readily-observable signature in the magnetic field above the ionosphere, namely an azimuthal field $B_{\varphi}$ that is added to the (dominantly) poloidal fields of the planet and ring current, which deflects field lines out of magnetic meridian planes. Physically, this deflection is caused by the ionospheric torque on the field line feet due to the ion-neutral collisions responsible for Pedersen conductance, and is such that for plasma sub-corotation the twist produces a positive $B_{\varphi}$ in the Southern Hemisphere, and a negative $B_{\varphi}$ in the Northern Hemisphere, thus producing a "lagging" field configuration. Application of Ampère's law in the axi-symmetric approximation yields

$B_{\varphi}=\mp \mu_{0} I_{h P}^{\prime} / \rho$,

where $I_{h P}^{\prime}$ is the Pedersen current per radian of azimuth at the feet of the field lines in question, and $\rho$ is the perpendicular distance from the rotation and magnetic axis. The upper and lower signs in Eq. (4) are appropriate to the Northern and Southern Hemispheres, respectively. The azimuthal field thus varies along each field line inversely with $\rho$ in the region between the ionospheric Pedersen current layer and where the field-aligned current closes across field lines in the outer magnetosphere. The $B_{\varphi}$ field produced by the CBO model was not calculated by Cowley et al. (2004b), but is shown in Fig. 3d evaluated just above the model ionosphere, the negative values for the Northern Hemisphere (dashed) being for convenience reversed in sign. The magnitude peaks at $\sim 100 \mathrm{nT}$ on open field lines at the openclosed field line boundary, and falls sharply to smaller values in the outer magnetosphere as the plasma angular velocity rises towards corotation. This fall in $B_{\varphi}$ is the magnetic signature of the upward-directed field-aligned current flowing in the boundary. A lower peak in $B_{\varphi}$ then also occurs in the sub-corotating middle magnetosphere region at larger co-latitudes, rising and falling in association with the pattern of field-aligned currents in accordance with Ampère's law.

We now consider the upward-directed field-aligned current density in the model in relation to auroral acceleration and precipitation of magnetospheric electrons. Assuming that the magnetospheric source populations can be described as isotropic Maxwellians of density $N$ and thermal energy $W_{t h}$ (equal to $k T$ ), the maximum current density and energy flux that can be provided to the ionosphere without field-aligned electron acceleration are

$j_{\mid i 0}=e N\left(\frac{W_{t h}}{2 \pi m_{e}}\right)^{1 / 2}$

and

$E_{f 0}=2 N W_{t h}\left(\frac{W_{t h}}{2 \pi m_{e}}\right)^{1 / 2}$.

These quantities correspond to the case of a full downwardgoing loss-cone and an empty upward-going loss-cone, and are equivalent to the quantities $j_{\| e}$ and $E_{f} e$ computed from the observed electron distributions in Fig. 2 on the assumption of near-isotropy. If, however, the required upward current density is larger than $j_{\| i 0}$, a field-aligned voltage must then be present to accelerate the magnetospheric electrons into the ionosphere. According to Knight's (1973) theory, the minimum field-aligned voltage which must be present is

$\Phi_{\| \min }=\frac{W_{t h}}{e}\left[\left(\frac{j_{\| i}}{j_{\| i 0}}\right)-1\right]$,

this value being appropriate if the "top" of the voltage drop is located at a radial distance well above the minimum value given by

$\left(\frac{r_{\min }}{R_{i}}\right) \approx\left(\frac{j_{\| i}}{j_{\| i 0}}\right)^{1 / 3}$,

where $R_{i}$ is the radial distance of the ionospheric current layer. The enhanced precipitating electron energy flux corresponding to Eq. (6) is then

$E_{f}=\frac{E_{f 0}}{2}\left[\left(\frac{j_{\| i}}{j_{\| i 0}}\right)^{2}+1\right]$,

following Lundin and Sandahl (1978).

The source parameters employed in the CBO model, based on Voyager electron data, are given in Table 1, together with the corresponding values of $j_{\| i 0}$ and $E_{f 0}$. The magnetosheath and outer magnetosphere parameters are applied to the upward current at the open-closed field line boundary, with magnetosheath parameters taken to apply poleward of the boundary and outer magnetosphere parameters 

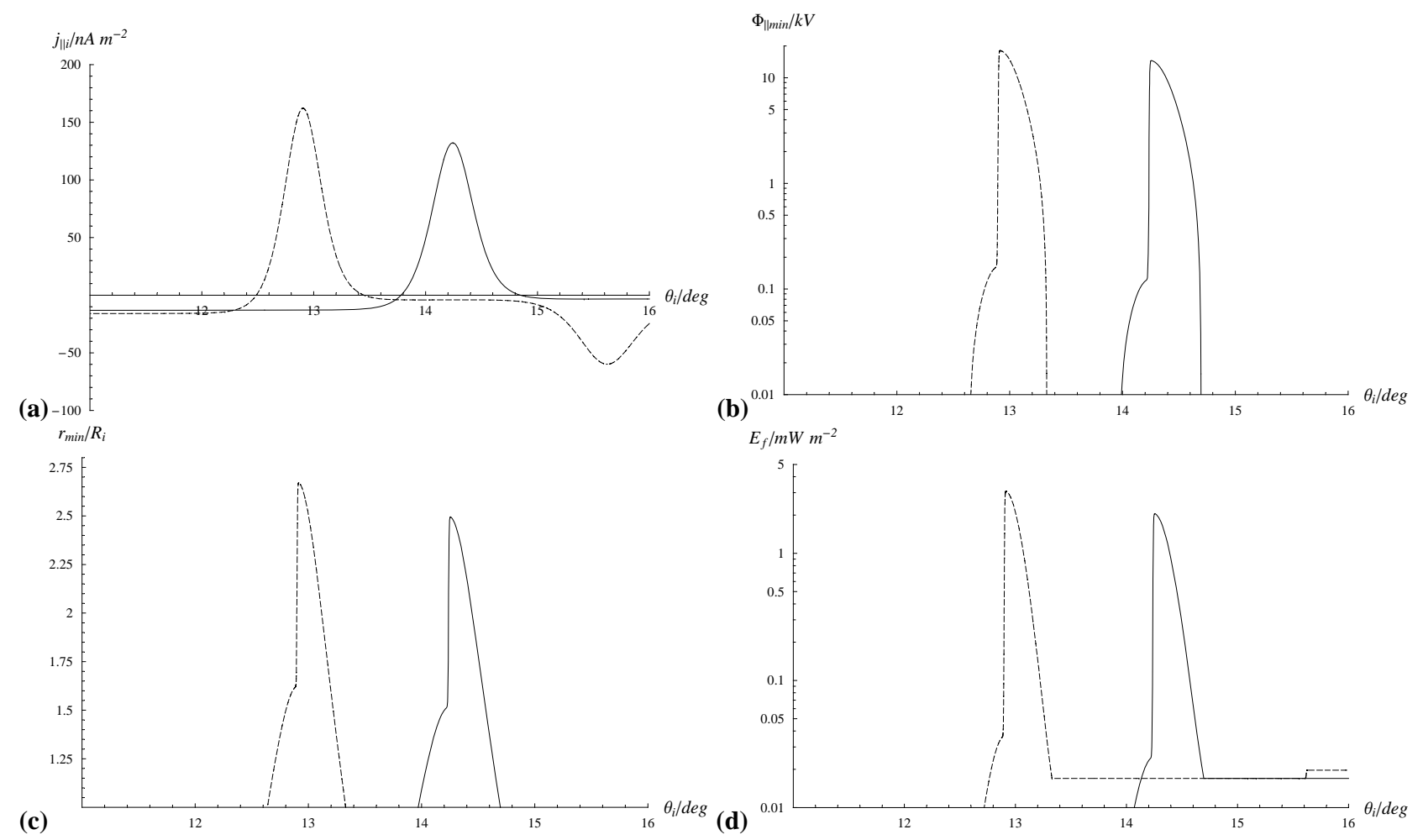

Fig. 4. Auroral acceleration parameters near the open-closed field line boundary for the CBO model plotted versus co-latitude $\theta_{i}$ in the ionosphere on an expanded scale, for both Northern (dashed lines) and Southern Hemispheres (solid lines). The panels show (a) the fieldaligned current density as in Fig. 3, (b) the minimum field-aligned voltage $\Phi_{\| \text {min }}$ required to produce this current density, given by Eq. (6) with electron source parameters shown in Table 1, (c) the corresponding precipitating electron energy flux given by Eq. (8), and (d) the minimum height of the electron acceleration region given by Eq. (7) in terms of the radial distance of the ionospheric Pedersen layer $R_{i}$ (equal to $R_{S}$ in a first approximation). Note that magnetosheath source parameters are employed poleward of the open-closed field line boundary (essentially where the current density peaks), and outer magnetosphere source parameters equatorward of the boundary.

equatorward of the boundary, switching sharply at the centre of the current layer where $F=F_{O}$ in Eq. (1). The middle magnetosphere parameters are then applied to the upward current region at larger co-latitudes on closed field lines. Comparison of the current densities in the latter region with the limiting middle magnetosphere current density of $\sim 65.7 \mathrm{nA} \mathrm{m}^{-2}$ shows that field-aligned electron acceleration is not required to carry these currents. Further, the unaccelerated energy flux of these electrons is sufficient to produce auroral emissions of only $\sim 0.2 \mathrm{kR}$ intensity, which is presently undetectable. However, the current density at the open-closed field-line boundary, peaking at $\sim 150 \mathrm{nA} \mathrm{m}^{-2}$ in the model, exceeds the limiting current densities for both magnetosheath $\left(\sim 38 \mathrm{nA} \mathrm{m}^{-2}\right)$ and outer magnetospheric $\left(\sim 8.5 \mathrm{nA} \mathrm{m}^{-2}\right)$ source electrons, thus implying that both source populations must be accelerated along the field lines to carry current densities of this magnitude. The accelerations required and the resulting energy fluxes are shown in Fig. 4, where we now focus on the vicinity of the open-closed field line boundary. Figure $4 \mathrm{a}$ thus repeats the current density curves but now on an expanded co-latitude scale, while Fig. $4 \mathrm{~b}$ shows the corresponding minimum fieldaligned voltage given by Eq. (6), where the step in the plots at the centre of the northern and southern upward current layers corresponds to the position where we switch from magnetosheath source parameters poleward of the centre to outer magnetosphere parameters equatorward of the centre, as previously indicated. It can be seen that the acceleration voltages are $\sim 100 \mathrm{~V}$ in the former case and $\sim 10 \mathrm{kV}$ in the latter. The corresponding precipitating energy fluxes obtained from Eq. (8) are shown in Fig. 4c. The values in the region where the current is assumed to be carried by magnetosheath electrons are of order $\sim 0.01 \mathrm{~mW} \mathrm{~m}^{-2}$, again resulting in currently undetectable UV emissions of $\sim 0.1 \mathrm{kR}$. However, in the region where the current is assumed carried by outer magnetospheric electrons the energy flux peaks above $\sim 1 \mathrm{~mW} \mathrm{~m}^{-2}$, resulting in auroral emissions in excess of $\sim 10 \mathrm{kR}$. On this basis Cowley et al. (2004b) concluded that Saturn's main oval is likely associated with the boundary between open and closed field lines, specifically with the region where the upward field-aligned current is carried by accelerated outer magnetosphere electrons. In Fig. $4 d$ we 
then show the minimum height of the acceleration region, obtained from Eq. (7). It can be seen that while the acceleration region for magnetosheath electrons can occur quite close to the planet, outer magnetospheric electrons must be accelerated at radial distances in excess of $\sim 2.5 R_{S}$.

\section{Comparison with Cassini data for Revolution 37}

We now consider the behaviour of the plasma and field parameters at Cassini on Rev 37 expected on the basis of the CBO model, obtained by mapping the model parameters along magnetic field lines between the ionosphere and the spacecraft using the same internal plus ring current field model as employed in Figs. 1 and 2. In Fig. 5a we first plot the co-latitude of the magnetic footprint of the spacecraft in the southern ionosphere versus UT, over the same $42 \mathrm{~h}$ interval as shown in Fig. 2. The corresponding model parameter values over the same interval are then shown in subsequent panels by the dot-dashed lines, while the solid lines in these panels correspond to a revised model that will be discussed later in Sect. 5. Comparison with Fig. 3 then shows that near the beginning of the interval the spacecraft was located inside the model region of open field lines at $\sim 8^{\circ}-9^{\circ}$ Southern Hemisphere co-latitude, and that it then passed across the model open-closed field line boundary at $\sim 14.2^{\circ}$ into the outer magnetosphere at $\sim 02: 00$ UT on 17 January. The spacecraft then remained within the model outer magnetosphere for the rest of the interval shown, eventually crossing the equatorial plane near the inner boundary of the outer magnetosphere at a radial distance of $\sim 15 R_{S}$ late in the second half of 17 January. These locations are then reflected in the behaviour of the model angular velocity shown in Fig. 5b (dot-dashed line), whose essential feature is the increase in normalised angular velocity from 0.3 to 0.8 as the spacecraft crossed from model open field lines to closed outer magnetosphere field lines. The conjugate equatorward Pedersen current per radian of azimuth in the model is displayed in Fig. 5c, showing steady growth as the open-closed fieldline boundary is approached, and a subsequent rapid drop on closed field lines as the angular velocity increases towards rigid corotation. The latter drop in ionospheric horizontal current is then coincident with the layer of intense upwarddirected field-aligned current just above the conjugate ionosphere shown in Fig. 5d, together with the related enhanced precipitating electron energy flux shown in Fig. 5e that produces the main auroral oval in this model. In Fig. $5 \mathrm{f}$ and $\mathrm{g}$ we then show the local field-aligned current density at the spacecraft $j_{\|}$, mapped along field lines from the ionosphere using $\left(j_{\|} / B\right)=$ constant, and the associated local azimuthal perturbation field $B_{\varphi}$ calculated from Eq. (4).

From Fig. 5 it is evident that the potential key observables are the plasma angular velocity, the field-aligned current density, and the azimuthal field perturbation in situ at the spacecraft, together with the auroral emission at the feet of the field lines. However, of the in situ parameters, plasma velocities are difficult to determine from the ion data obtained, and are not presently available. In addition, it can be seen that the model upward-directed field-aligned current density at the spacecraft peaks at $\sim 40 \mathrm{pA} \mathrm{m}^{-2}$ at the openclosed field line boundary, a value that is typically $\sim 0.1 \%$ of the current associated with the in situ electron flux moving in one direction along the field lines, shown in the fourth panel of Fig. 2. The local field-aligned current is thus essentially unobservable directly in electron fluxes, as already mentioned in Sect. 3. However, the related in situ azimuthal magnetic field is distinctly observable, growing to peak positive values in the model of $\sim 2 \mathrm{nT}$ on open field lines near the open-closed field line boundary, and then dropping to smaller values in the outer magnetosphere due to the reduced ionospheric torque as the plasma angular velocity rises, and in association with the related layer of upward-directed fieldaligned current at the boundary. The observations appropriate to the model thus centre on the behaviour of the azimuthal field at the open-closed field line boundary, and its relation to the precipitation of accelerated electrons forming the main auroral oval.

Thus comparing the key results of the $\mathrm{CBO}$ model shown by the dot-dashed lines in Fig. 5 with the data in Fig. 2, plotted over the same interval, it is clear that the model and the data show the same qualitative behaviour. Positive $B_{\varphi}$ was consistently observed by Cassini in the region of open field lines, which then fell to small values in the outer magnetosphere across the cusp open-closed field line boundary, indicative of a major layer of upward-directed field-aligned current flowing in the boundary, with the main UV oval lying at the feet of these field lines. As pointed out by Bunce et al. (2008b), these data thus provide significant support for the conclusion of Cowley et al. (2004a, b) that Saturn's auroral oval is associated with a strong shear in rotational flow across the boundary between open and closed field lines, and its associated layer of upward-directed field-aligned current. These data do not support the suggestion of Sittler et al. (2006) that the aurora are formed on closed field lines inside the magnetosphere near the outer-middle magnetosphere boundary, the latter usually mapping near $\sim 15 R_{S}$ in the equatorial plane and to $\sim 16^{\circ}-18^{\circ}$ in the southern ionosphere (e.g. Bunce et al., 2008a). Indeed, as can be seen from the position data in Figs. 1 and 2, the spacecraft only approached such field lines from higher latitudes in the second half of day 17 during its south to north passage through the dayside equatorial region, after the interval examined here and shown in Fig. 2, when the spacecraft was clearly located equatorward of the auroral oval. Still less do these data support the notion of an auroral connection with corotation breakdown in the central middle magnetosphere.

Although qualitative agreement with the $\mathrm{CBO}$ model is thus apparent, it can also be seen from Figs. 2 and 5 that the model and data differ quantitatively in three significant respects. The first is that the model current layer occurs 

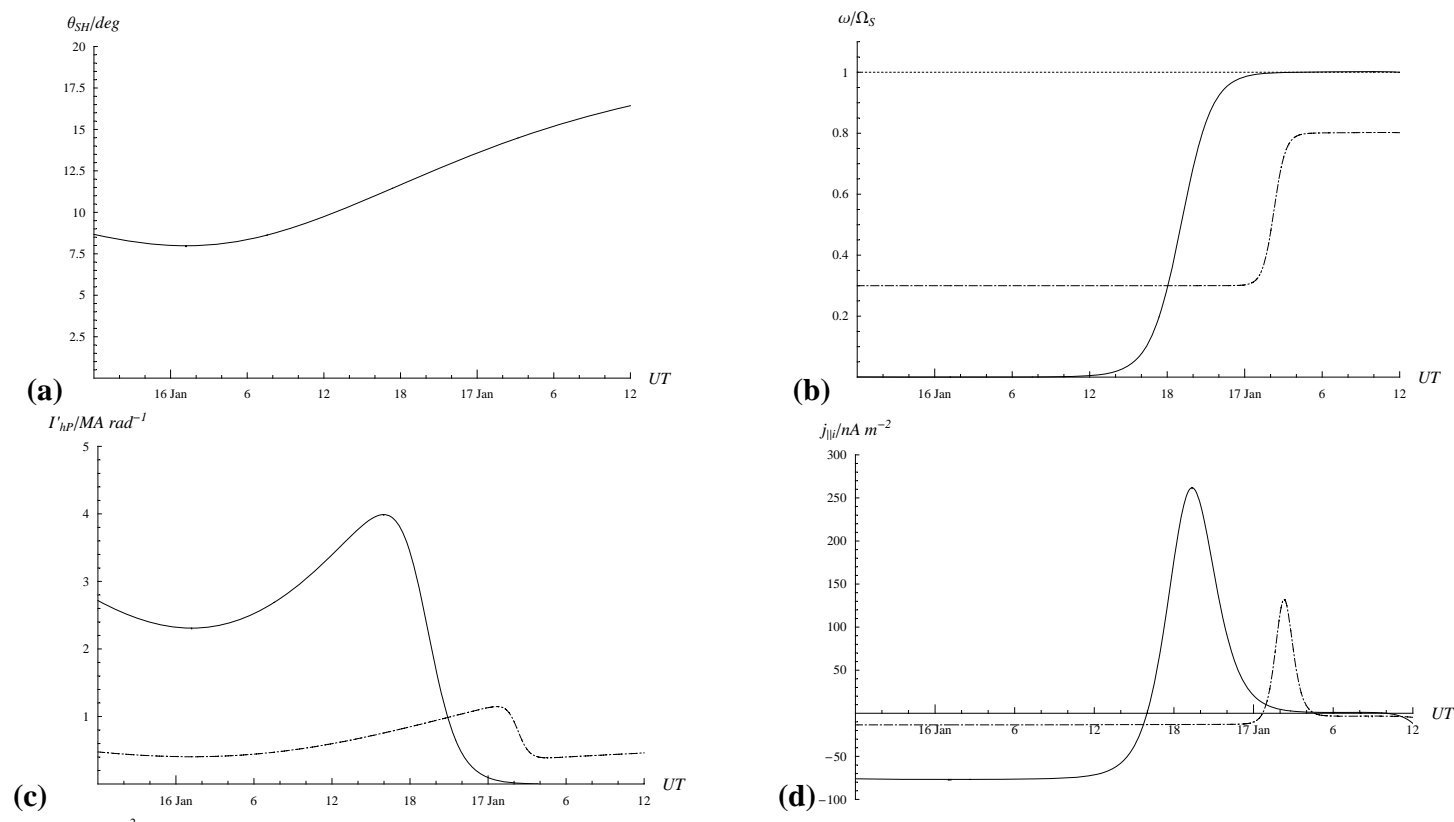

(c) ${ }_{E_{f} / m W m^{-2}}$

(d)
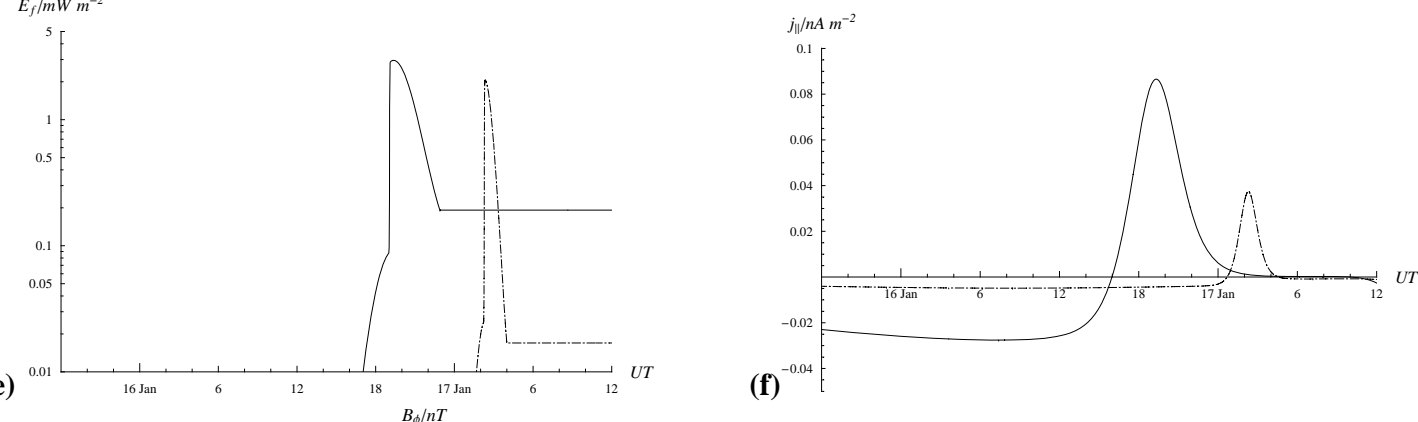

(g)

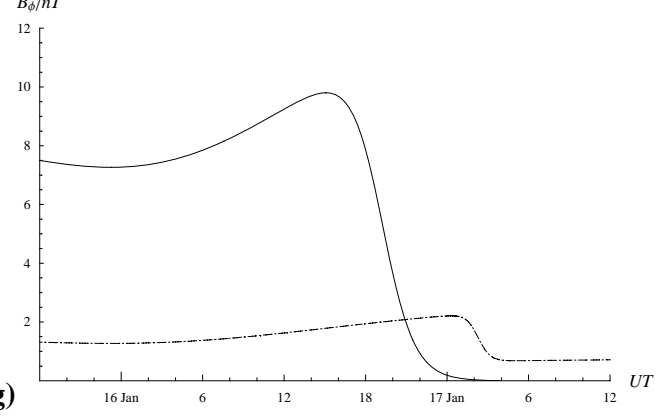

Fig. 5. Model parameters are shown mapped along Southern Hemisphere field lines to Cassini using the same magnetic model as employed in Figs. 1 and 2, plotted versus UT over the same $42 \mathrm{~h}$ interval of Rev 37 as shown in Fig. 2. Panel (a) first shows the co-latitude of the spacecraft mapped magnetically in the southern ionosphere. In subsequent panels the dot-dashed lines correspond to the CBO model shown in Figs. 3 and 4, while the solid lines correspond to the revised model shown in Figs. 6 and 7. These panels show (b) the plasma angular velocity $\omega$ normalised to the planet's angular velocity $\Omega_{S}$, where the horizontal dotted line represents rigid corotation, (c) the equatorwarddirected horizontal ionospheric Pedersen current per radian of azimuth $I_{h P}^{\prime}$ in the conjugate ionosphere, (d) the field-aligned current density just above the conjugate ionosphere $j_{\| i}$, where positive and negative values indicate upward- and downward-directed currents, respectively, (e) the energy flux of precipitating electrons in the conjugate southern ionosphere, those within the upward-directed field-aligned current layer being enhanced by field-aligned potentials compared with the source populations given in Table 1 , (f) the field-aligned current density in situ at the spacecraft $j_{\|}$, mapped along field lines from the ionosphere using $\left(j_{\|} / B\right)=$ constant, and (g) the azimuthal magnetic field $B_{\varphi}$ at the spacecraft produced by the combined Pedersen and field-aligned current system, given by Eq. (4). 
somewhat later in time in Fig. 5 than in the data in Fig. 2, meaning that it is located at slightly too large a co-latitude, depending on the amount of open flux that happened to be present in the polar cap at the time. The observed boundary and auroral oval were centred near $\sim 12^{\circ}$ in the Southern Hemisphere rather than the model value of $\sim 14^{\circ}$. This difference lies well within the usual range of oval variability at Saturn (Badman et al., 2005, 2006). Second, it can be seen in Fig. 2 that the observed behaviour of $B_{\varphi}$ is not quasimonotonic in the boundary region, but shows an initial fall near $\sim 12: 00$ UT on 16 January, followed by a subsequent major rise and fall centred near $\sim 18: 00$ UT. On a strictly spatial interpretation this would require the presence of a major layer of upward-directed field-aligned current where $B_{\varphi}$ initially falls, followed by comparable or larger downward and upward current layers on either side of the subsequent $B_{\varphi}$ peak, driven by a layered pattern of strongly subcorotating flow where $B_{\varphi}$ is large, and near-corotating flow where $B_{\varphi}$ is small. Physically, this represents unexpected and unlikely behaviour, in addition to which there is no evidence in Figs. 1 or 2 for two individual peaks in the conjugate UV emission that would correspond to the two comparable but spatially separated layers of upward-directed fieldaligned current. It thus seems most likely that the observations in Fig. 2 correspond to a single current layer that oscillates across the spacecraft, probably related to the planetary period oscillations in field and plasma that are ubiquitous in Saturn's magnetosphere (e.g. Cowley et al., 2006; Gurnett et al., 2007; Southwood and Kivelson, 2007; Andrews et al., 2008; Nichols et al., 2008). This is the hypothesis that will be adopted here, while remaining a topic for future investigation.

The third difference between the CBO model results in Fig. 5 and the Cassini data in Fig. 2, and the most significant, is that the magnitude of the observed azimuthal field on open field lines is typically $\sim 5$ times larger than that of the model, peaking in the boundary region at $\sim 10 \mathrm{nT}$ instead of $\sim 2 \mathrm{nT}$ in the model. Taken together with the small negative values of $B_{\varphi}$ observed in the outer magnetosphere region (implying slight plasma super-corotation in terms of the model), the implication is that the total field-aligned current flowing in the boundary layer is significantly larger than that in the model, $\sim 4-5 \mathrm{MA} \mathrm{rad}^{-1}$ as shown by Bunce et al. (2008) using Eq. (4), rather than $\sim 0.8 \mathrm{MA} \mathrm{rad}^{-1}$ in the model (Fig. 3b). Combined with Bunce et al.'s (2008) estimate of the layer width of $\sim 1.5^{\circ}$ in co-latitude, wider than in the CBO model by a factor of $\sim 3$, this implies fieldaligned current densities just above the top of the atmosphere peaking near $\sim 200-300 \mathrm{nA} \mathrm{m}^{-2}$, somewhat larger than the peak Southern Hemisphere field-aligned current density of $\sim 130 \mathrm{nA} \mathrm{m}^{-2}$ in the CBO model (Figs. $3 \mathrm{c}$ and $4 \mathrm{a}$ ).

\section{Modified magnetosphere-ionosphere coupling model for Saturn}

Following the above discussion, we thus propose parameter adjustments to the $\mathrm{CBO}$ model that bring better agreement with the Rev 37 data. The first simple change is to relocate the open-closed field line boundary to $\sim 12^{\circ}$ in the Southern Hemisphere (thus most closely representing auroral conditions for the slightly contracted oval in image B), by making the revised choice $F_{O}=1100 \mathrm{nT} R_{S}^{2}$ in Eq. (1). We also make the current layer in the boundary wider, as suggested by the analysis of Bunce et al. (2008b), by the choice $\Delta F_{O}=140 \mathrm{nT} R_{S}^{2}$. In order to make the total upward current larger by factors of $\sim 5$ as required by the observed $B_{\varphi}$ fields, there are two possible choices. The first is to increase the effective height-integrated ionospheric Pedersen conductivity in Eq. (2) whose value is uncertain as noted above, while the second is to increase the departure of the plasma flow from rigid corotation on open field lines. However, since plasma anti-corotation on open field lines is not physically plausible, we set the minimum realistic value $\left(\omega / \Omega_{S}\right)_{O}=0$ in Eq. (1), such that the open field lines do not rotate at all in the inertial frame. We also assume that the plasma nearrigidly corotates on outer magnetosphere closed field lines, i.e. $\left(\omega / \Omega_{S}\right)_{O M}=1.0$ in Eq. (1). We note that the observations in Fig. 2 indicate small negative $B_{\varphi}$ values in this region implying weak super-corotation of the plasma, however this again seems somewhat inappropriate for a model intended to represent near-steady state conditions. In order to match the $B_{\varphi}$ fields observed on open field lines we then require to increase the effective Pedersen conductivity to $\Sigma_{P}^{*}=4$ mho, noting as above the southern summer conditions that prevailed during this period, with the southern pole being tilted by $\sim 14^{\circ}$ towards the Sun. The other model parameters in Eq. (1) defining the angular velocity profile in the middle magnetosphere region and its interface with the outer magnetosphere remain unmodified, since as indicated above, Cassini did not enter these regions on Rev 37, and hence did not provide additional information that could inform revised choices.

Revised model values using the above parameters are shown plotted versus co-latitude in the ionosphere in Fig. 6, in the same format as Fig. 3. For simplicity, the same effective Pedersen conductivity of 4 mho is employed in the results shown for both hemispheres, though as indicated above, the conductivity of the Northern Hemisphere could be significantly reduced compared with the southern under the northern winter conditions prevailing. This would have the effect of reducing the currents in the north compared with the south, together with the accelerating voltages and precipitating electron energy fluxes. The model curves in Fig. 6 show similar basic patterns as Fig. 3, but the Pedersen currents are enhanced by factors of $\sim 4$, and thus so too is the magnitude of the azimuthal field just above the Pedersen layer, which now peaks at $\sim 500 \mathrm{nT}$ just inside the boundary of 

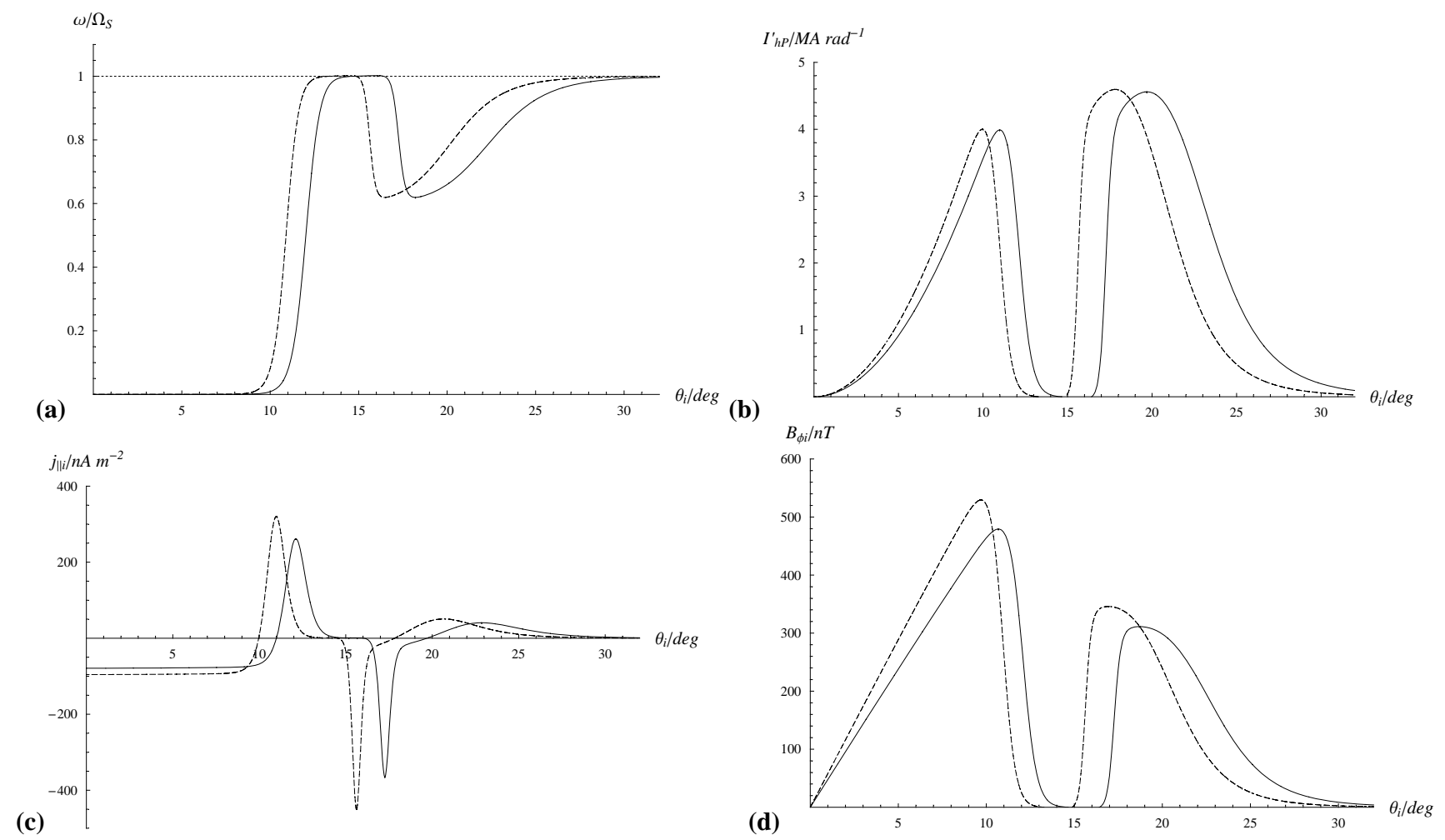

Fig. 6. As for Fig. 3, but now for the revised Saturn magnetosphere-ionosphere coupling current model discussed in Sect. 5. In particular the effective ionospheric Pedersen conductivity has been increased to 4 mho in both hemispheres.

open field lines, and at $\sim 300 \mathrm{nT}$ in the outer middle magnetosphere, assuming that the same elevated ionospheric Pedersen conductivity applies uniformly throughout. The fall in the Pedersen current across the open-closed boundary is now $\sim 4 \mathrm{MA} \mathrm{rad}^{-1}$, equal to the total upward field-aligned current per radian of azimuth flowing in the boundary, a factor of $\sim 5$ larger than the $\sim 0.8 \mathrm{MA} \mathrm{rad}^{-1}$ flowing in the CBO model. This increase results from the larger flow shear at the boundary (factor of two) and the larger Pedersen conductivity (factor of four), while being reduced somewhat by the reduced radius of the boundary. This in turn results in larger field-aligned current densities at the open-closed field boundary compared with the CBO model, but only by a factor of about two, because of the increased layer width employed in the revised model. The field-aligned currents on closed field lines at lower latitudes are also significantly elevated, with the downward current at the boundary between the outer and middle magnetosphere regions now becoming a significant feature, though one whose physical validity requires further study. We also note that the peak upward current density in the middle magnetosphere still remains less than the critical density for downward electron acceleration in this region, $\sim 66 \mathrm{nA} \mathrm{m}^{-2}$ as given in Table 1 .

In Fig. 7 we thus continue to focus on auroral acceleration at the open-closed field line boundary in the revised model, as in Fig. 4. In the revised model, however, we have also modified the electron source population parameters compared with the CBO model in light of the observed values shown in Fig. 2. While the previous magnetosheath parameters employed still seem appropriate in this case, the outer magnetosphere parameters (at least as applicable to Rev 37) need to be revised upward in both density and temperature. Here we use $N=0.04 \mathrm{~cm}^{-3}$ and $W_{t h}=2 \mathrm{keV}$ in Eq. (5) to obtain a new limiting current density of $\sim 48 \mathrm{nA} \mathrm{m}^{-2}$ in this region, and a new limiting energy flux of $\sim 0.2 \mathrm{~mW} \mathrm{~m}^{-2}$, as also given in the outer magnetosphere column of Table 1 . We note, however, that this elevated value of the outer magnetosphere limiting current density still falls considerably short of the peak ionospheric field-aligned current density in the open-closed boundary, shown on an expanded co-latitude scale in Fig. 7a (as anticipated in the discussion of Fig. 2 in Sect. 3). Thus as in the original CBO model, acceleration of magnetospheric source electrons at the open-closed boundary is required whether they originate from magnetosheath or outer magnetosphere sources. The field-aligned voltage profiles displayed in Fig. $7 \mathrm{~b}$ show acceleration over a broader co-latitude region than in Fig. $4 \mathrm{~b}$, now peaking at $\sim 300 \mathrm{~V}$ where the current is taken to be carried by magnetosheath electrons poleward of the open-closed field line boundary (where the current density peaks), but still near $\sim 10 \mathrm{kV}$ in 

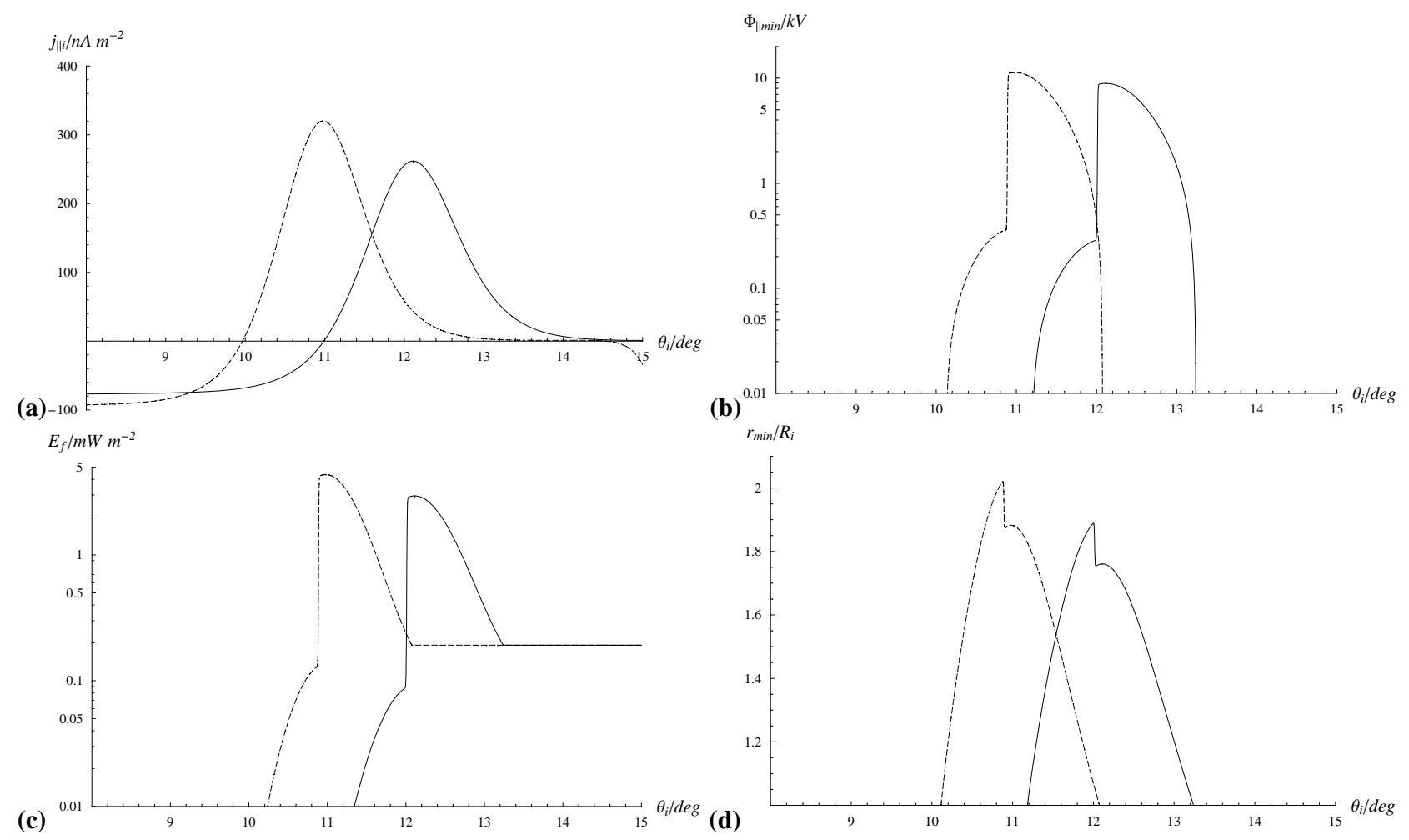

(c)

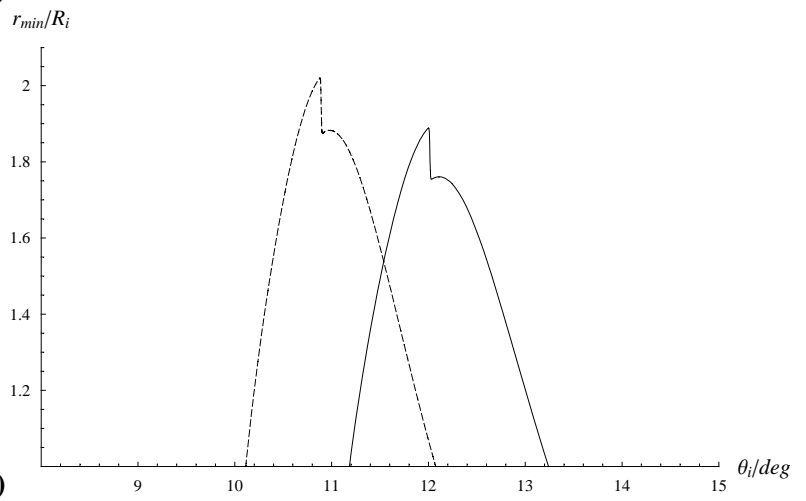

Fig. 7. As for Fig. 4, but now for the revised Saturn magnetosphere-ionosphere coupling current model discussed in Sect. 5.

the region where the current is taken to be carried by outer magnetosphere electrons. The resulting precipitating energy fluxes shown in Fig. 7c are again weak where the current is carried by accelerated magnetosheath electrons, peaking at $\sim 0.1 \mathrm{~mW} \mathrm{~m}^{-2}$, thus producing UV emissions of only $\sim 1 \mathrm{kR}$. However, the precipitating energy flux exceeds $\sim 1 \mathrm{~mW} \mathrm{~m}^{-2}$ in the region where the current is carried by outer magnetosphere electrons, thus again resulting in the formation of an auroral oval with UV emission intensities of a few tens of $\mathrm{kR}$, which is now somewhat wider than in the CBO model. We also note that the horizontal lines extending to larger colatitudes in this plot represent the precipitating energy flux of the unaccelerated outer magnetosphere source electrons, which as indicated above is $\sim 0.2 \mathrm{~mW} \mathrm{~m}^{-2}$ according to the revised model values (see also Fig. 2). This is then capable of producing $\mathrm{UV}$ emission of $\sim 2 \mathrm{kR}$ intensity in a region extending a few degrees equatorward of the main oval mapping to the outer magnetosphere, if particle pitch-angle scattering is sufficient to maintain a full loss cone. Under favourable circumstances this emission might be detectable as a lowintensity "halo" extending equatorward of the main oval located at the open-closed boundary. Figure $7 \mathrm{~d}$ then shows that the auroral acceleration regions in this case must form at radial distances exceeding $\sim 2 R_{S}$, a smaller limiting distance than in the CBO model for the principal outer magnetosphere source region, due to the higher number flux of the source population.
We finally return to Fig. 5, where the parameters of the revised model are mapped along model field lines to the Cassini spacecraft as for the CBO model, and are shown by the solid lines. Here the critical feature is the revised $B_{\varphi}$ signature at the spacecraft shown in Fig. $5 \mathrm{~g}$, which is increased by a factor of about four compared with the CBO model, and peaks at $\sim 10 \mathrm{nT}$ near the revised boundary of open and closed field lines in good overall agreement with the observed values in Fig. 2. This, taken together with the related precipitating electron energy flux profile in Fig. 5e, showing the formation of a UV auroral oval at the feet of the boundary field lines with peak intensities of $\sim 30 \mathrm{kR}$, completes our demonstration of the overall agreement between the revised magnetosphere-ionosphere coupling model presented here with the observations in Fig. 2. The model oval is somewhat narrower and brighter than the observed oval emissions along the spacecraft track plotted in the bottom panel of Fig. 2 (see also Fig. 1), but as discussed above in Sect. 3, the latter will have been somewhat spread in colatitude by instrument resolution and projection effects, possibly by factors of $2-3$.

\section{Summary and conclusions}

The first coordinated Cassini observations on southern highlatitude field lines in Saturn's magnetosphere combined with 
imaging of the southern polar UV emissions by the HST has shown that the main oval at noon is associated with a major layer of upward-directed field-aligned current spanning the cusp boundary between open and closed field lines (Bunce et al., 2008b). These findings thus support the conclusions drawn from prior theoretical discussion by Cowley et al. (2004a) and the subsequent quantitative modelling of Cowley et al. (2004b) (the "CBO" model), that associate Saturn's auroral oval with flow shear and field-aligned currents at the open-closed field line boundary. Given the qualitative correspondence between these observations and the CBO model, we have therefore made a detailed quantitative comparison, showing that agreement requires the presence both of a major shear in azimuthal flow at the boundary, together with an effective ionospheric Pedersen conductivity that is larger than that previously employed by a factor of about four (i.e. a conductivity of $\sim 4$ mho, compared with $\sim 1 \mathrm{mho}$ ). We note that this enhanced conductivity applies to the southern ionosphere under summer conditions. These factors increase the total upward-directed field-aligned current per radian of azimuth flowing in the model open-closed field line boundary by a factor of about five compared with the CBO model, from $\sim 0.8 \mathrm{MA} \mathrm{rad}^{-1}$ to $\sim 4 \mathrm{MA} \mathrm{rad}^{-1}$, in accordance with the observations. This also results in increased field-aligned current densities in the boundary by a factor of about two, offset by an increased width of the layer suggested by the Cassini data. The Cassini data also suggest that the electron number flux in the outer magnetosphere region that forms the primary source of auroral electrons is significantly larger than that employed in the CBO model based on Voyager data, but nevertheless the model current densities still require acceleration of these electrons along the field lines through $\sim 10 \mathrm{kV}$ voltages. The precipitating energy flux of the accelerated electrons is then sufficient to produce UV emissions of a few tens of kR intensity, similar to those observed by the HST in the main oval. The unaccelerated outer magnetosphere electrons may also provide a "halo" of weak few-kR UV emissions extending equatorward of the main oval by a few degrees, provided these electrons are sufficiently pitch-angle scattered that their loss cone is maintained full.

While thus providing support for the view that Saturn's main oval emissions map to the open-closed field line boundary, the data discussed here do not support the idea that Saturn's aurora map to the corotation breakdown region in the middle magnetosphere as they do at Jupiter, nor the theoretical discussion of Sittler et al. (2006) that places the oval inside the magnetosphere on closed field lines mapping typically to $\sim 15 R_{S}$ in the equatorial plane, corresponding to the vicinity of the boundary between outer and middle magnetosphere field lines. Of course our particular results relate specifically to the vicinity of the dayside cusp near to noon. However, as in the present case shown in Fig. 1, Saturn's oval in the vicinity of noon is generally either continuous with or connected to the oval at other local times (see e.g.
Gérard et al., 2005), thus suggesting a common or directly related origin. It will thus be of interest in future analysis of Cassini data to determine whether the major layer of upwarddirected field-aligned current that has been related here to the main oval emissions is also present at the open-closed field line boundary at other local times.

Acknowledgements. S. W. H. Cowley was supported by STFC grant PP/D002117/1 and a Royal Society Leverhulme Trust Senior Research Fellowship, and E. J. Bunce and D. L. Talboys by STFC grant PP/E001130/1. C. S. Arridge, A. J. Coates, and ELS data processing by L. K. Gilbert and G. R. Lewis were supported by the STFC rolling grant to MSSL/UCL. J.-C. Gérard and D. Grodent were supported by the Belgian Fund for Scientific Research (FNRS), and by the PRODEX Program managed by the European Space Agency in collaboration with the Belgian Federal Science Policy Office. Work at Boston was supported by grant HST-GO10862.01-A from the Space Telescope Science Institute to Boston University. This study employs observations using the NASA/ESA Hubble Space Telescope, obtained at the Space Telescope Science Institute, which is operated by AURA for NASA.

Topical Editor I. A. Daglis thanks two anonymous referees for their help in evaluating this paper.

\section{References}

Andrews, D. J., Bunce, E. J., Cowley, S. W. H., Dougherty, M. K., Provan, G., and Southwood, D. J.: Planetary period oscillations in Saturn's magnetosphere: Phase relation of equatorial magnetic field oscillations and SKR modulation, J. Geophys. Res., 113, A09205, doi:10.1029/2007JA012937, 2008.

Arridge, C. S., Achilleos, N., Dougherty, M. K., Khurana, K. K., and Russell, C. T.: Modeling the size and shape of Saturn's magnetopause with variable dynamic pressure, J. Geophys. Res., 111, A11227, doi:10.1029/2005JA011574, 2006.

Badman, S. V., Bunce, E. J., Clarke, J. T., Cowley, S. W. H., Gérard, J.-C., Grodent, D., and Milan, S. E.: Open flux estimates in Saturn's magnetosphere during the January 2004 CassiniHST campaign, and implications for reconnection rates, J. Geophys. Res., 110, A11216, doi:10.1029/2005JA011240, 2005.

Badman, S. V., Cowley, S. W. H., Gérard, J.-C., and Grodent, D.: A statistical analysis of the location and width of Saturn's southern auroras, Ann. Geophys., 24, 3533-3545, 2006, http://www.ann-geophys.net/24/3533/2006/.

Bunce, E. J., Cowley, S. W. H., Alexeev, I. I., Arridge, C. S., Dougherty, M. K., Nichols, J. D., and Russell, C. T.: Cassini observations of the variation of Saturn's ring current parameters with system size, J. Geophys. Res., 112, A10202, doi:10.1029/2007JA012275, 2007.

Bunce, E. J., Arridge, C. S., Cowley, S. W. H., and Dougherty, M. K.: Magnetic field structure of Saturn's dayside magnetosphere and its mapping to the ionosphere: Results from ring-current modelling, J. Geophys. Res., 113, A02207, doi:10.1029/2007JA012538, 2008a.

Bunce, E. J., Arridge, C. S., Clarke, J. T., Coates, A. J., Cowley, S. W. H., Dougherty, M. K., Gérard, J.-C., Grodent, D., Hansen, K. C., Nichols, J. D., Southwood, D. J., and Talboys, D. L.: Origin of Saturn's aurora: Simultaneous observations by Cassini and 
the Hubble Space Telescope, J. Geophys. Res., 113, A09209, doi:10.1029/2008JA013257, 2008b.

Clarke, J. T., Ballester, G., Trauger, J., Ajello, J., Pryor, W., Tobiska, K., Connerney, J. E. P., Gladstone, G. R., Waite Jr., J. H., Ben Jaffel, L., and Gérard, J.-C.: Hubble Space Telescope imaging of Jupiter's UV aurora during the Galileo orbiter mission, J. Geophys. Res., 103, 20 217-20 236, 1998.

Clarke, J. T., Gérard, J.-C., Grodent, D., Wannawichian, S., Gustin, J., Connerney, J., Crary, F., Dougherty, M., Kurth, W., Cowley, S. W. H., Bunce, E. J., Hill, T., and Kim, J.: Morphological differences between Saturn's ultraviolet aurorae and those of Earth and Jupiter, Nature, 433, 717-719, 2005.

Cowley, S. W. H. and Bunce, E. J.: Origin of the main auroral oval in Jupiter's coupled magnetosphere-ionosphere system, Planet. Space Sci., 49, 1067-1088, 2001.

Cowley, S. W. H. and Bunce, E .J.: Corotation-driven magnetosphere-ionosphere coupling currents in Saturn's magnetosphere and their relation to the auroras, Ann. Geophys., 21, 1691-1707, 2003, http://www.ann-geophys.net/21/1691/2003/.

Cowley, S. W. H., Bunce, E. J., and Prangé, R.: Saturn's polar ionospheric flows and their relation to the main auroral oval, Ann. Geophys., 22, 1379-1394, 2004a, http://www.ann-geophys.net/22/1379/2004/.

Cowley, S. W. H., Bunce, E. J., and O'Rourke, J. M.: A simple quantitative model of plasma flows and currents in Saturn's polar ionosphere, J. Geophys. Res., 109, A05212, doi:10.1029/2003JA010375, 2004b.

Cowley, S. W. H., Alexeev, I. I., Belenkaya, E. S., Bunce, E. J., Cottis, C. E., Kalegaev, V. V., Nichols, J. D., Prangé, R., and Wilson, F. J.: A simple axi-symmetric model of magnetosphereionosphere coupling currents in Jupiter's polar ionosphere, J. Geophys. Res., 110, A11209, doi:10.1029/2005JA011237, 2005.

Cowley, S. W. H., Wright, D. M., Bunce, E. J., Carter, A. C., Dougherty, M. K., Giampieri, G., Nichols, J. D., and Robinson, T. R.: Cassini observations of planetary-period magnetic field oscillations in Saturn's magnetosphere: Doppler shifts and phase motion, Geophys. Res. Lett., 33, L07104, doi:10.1029/2005GL025522, 2006.

Davis Jr., L. and Smith, E. J.: A model of Saturn's magnetic field based on all available data, J. Geophys. Res., 95, 15 257-15 261, 1990.

Dougherty, M. K., Kellock, S., Southwood, D. J., Balogh, A., Smith, E. J., Tsurutani, B. T., Gerlach, B., Glassmeier, K. H., Gleim, F., Russell, C. T., Erdos, G., Neubauer, F. M., and Cowley, S. W. H.: The Cassini magnetic field investigation, Space Sci. Rev., 114, 331-383, 2004.

Dougherty, M. K., Achilleos, N., André, N., Arridge, C. S., Balogh, A., Bertucci, C., Burton, M. E., Cowley, S. W. H., Erdos, G., Giampieri, G., Glassmeier, K. H., Khurana, K. K., Leisner, J., Neubauer, F. M., Russell, C. T., Smith, E. J., Southwood, D. J., and Tsurutani, B. T.: Cassini magnetometer observations during Saturn orbit insertion, Science, 307, 1266-1270, 2005.

Gérard, J.-C., Grodent, D., Gustin, J., Saglam, A., Clarke, J. T., and Trauger, J. T.: Characteristics of Saturn's FUV aurora observed with the Space Telescope Imaging Spectrograph, J. Geophys. Res., 109, A09207, doi:10.1029/2004JA010513, 2004.

Gérard, J.-C., Bunce, E. J., Grodent, D., Cowley, S. W. H.,
Clarke, J. T., and Badman, S. V.: Signature of Saturn's auroral cusp: Simultaneous HST FUV observations and upstream solar wind monitoring, J. Geophys. Res., 110, A11201, doi:10.1029/2005JA011094, 2005.

Grodent, D., Gérard, J.-C., Cowley, S. W. H., Bunce, E. J., and Clarke, J. T.: Variable morphology of Saturn's southern ultraviolet aurora, J. Geophys. Res., 110, A07215, doi:10.1029/2004JA010983, 2005.

Gurnett, D. A., Persoon, A. M., Kurth, W. S., Groene, J. B., Avenkamp, T. F., Dougherty, M. K., and Southwood, D. J.: The variable rotation period of the inner region of Saturn's plasma disk, Science, 316, 442-445, 2007.

Hill, T. W.: Inertial limit on corotation, J. Geophys. Res., 84, 6554 6558, 1979.

Hill, T. W.: The jovian auroral oval, J. Geophys. Res., 106, 81018107, 2001.

Isbell, J., Dessler, A. J., and Waite Jr., J. H.: Magnetospheric energization by interaction between planetary spin and the solar wind, J. Geophys. Res., 89, 10 716-10722, 1984.

Jackman, C. M. and Cowley, S. W. H.: A model of the plasma flow and current in Saturn's polar ionosphere under conditions of strong Dungey-cycle driving, Ann. Geophys., 24, 1029-1055, 2006,

http://www.ann-geophys.net/24/1029/2006/.

Knight, S.: Parallel electric fields, Planet. Space Sci., 21, 741-750, 1973.

Kurth, W. S., Lecacheux, A., Avenkamp, T. F., Groene, J. B., and Gurnett, D. A.: A Saturn longitude system based on a variable kilometric radiation period, Geophys. Res. Lett., 34, L02201, doi:10.1029/2006GL028336, 2007.

Lewis, G. R., Andre, N., Arridge, C. S., Coates, A. J., Gilbert, L. K., Linder, D. R., and Rymer, A. M.: Derivation of density and temperature from the Cassini-Huygens CAPS Electron Spectrometer, Planet. Space Sci., 56, 901-912, 2008.

Lundin, R. and Sandahl, I.: Some characteristics of the parallel electric field acceleration of electrons over discrete auroral arcs as observed from two rocket flights, in Symposium on European Rocket Research, ESA SP-135, ESA, Noordwijk, The Netherlands, p. 125, 1978.

Nichols, J. D., Clarke, J. T., Cowley, S. W. H., Duval, J., Farmer, A. J., Gérard, J.-C., Grodent, D., and Wannawichian, S.: The oscillation of Saturn's southern auroral oval, J. Geophys. Res., in press, doi:10.1029/2008JA013444, 2008.

Prangé, R., Rego, D., Pallier, L., Connerney, J. E. P., Zarka, P., and Queinnec, J.: Detailed study of FUV jovian auroral features with the post-COSTAR HST faint object camera, J. Geophys. Res., 103, 20 195-20 215, 1998.

Rego, D., Prangé, R., and Gérard, J.-C.: Lyman $\alpha$ and $\mathrm{H}_{2}$ bands from the giant planets: 1. Excitation by proton precipitation in the jovian aurorae, J. Geophys. Res., 99, 17 075-17 094, 1994.

Richardson, J. D.: Thermal ions at Saturn: Plasma parameters and implications, J. Geophys. Res., 91, 1381-1389, 1986.

Richardson, J. D. and Sittler Jr., E. C.: A plasma density model for Saturn based on Voyager observations, J. Geophys. Res., 95, 12 019-12 031, 1990.

Saur, J., Mauk, B. H., Kassner, A., and Neubauer, F. M.: A model for the azimuthal plasma velocity in Saturn's magnetosphere, J. Geophys. Res., 109, A05217, doi:10.1029/2003JA010207, 2004. 
Sittler Jr., E. C., Blanc, M. F., and Richardson, J. D.: Proposed model for Saturn's auroral response to the solar wind: centrifugal instability model, J. Geophys. Res., 111, A06208, doi:10.1029/2005JA011191, 2006.

Stallard, T. S., Miller, S., Cowley, S. W. H., and Bunce, E. J.: Jupiter's polar ionospheric flows: measured intensity and velocity variations poleward of the main auroral oval, Geophys. Res. Lett., 30(5), 1221, doi:10.1029/2002GL016031, 2003.

Stallard, T. S., Miller, S., Trafton, L. M., Geballe, T. R., and Joseph, R. D.: Ion winds in Saturn's southern auroral/polar region, Icarus, 167, 204-211, 2004.

Southwood, D. J. and Kivelson, M. G.: A new perspective concerning the influence of the solar wind on Jupiter, J. Geophys. Res., 106, 6123-6130, 2001.

Southwood, D. J. and Kivelson, M. G.: Saturn magnetospheric dynamics: Elucidation of a camshaft model, J. Geophys. Res., 112, A12222, doi:10.1029/2007JA012254, 2007.
Vasyliunas, V. M.: Plasma distribution and flow, in: Physics of the Jovian Magnetosphere, edited by: Dessler, A. J., Cambridge Univ. Press, Cambridge, UK, p. 395-453, 1983.

Young, D. T., Berthelier, J. J., Blanc, M., Burch, J. L., Coates, A. J., Goldstein, R., Grande, M., Hill, T. W., Johnson, R. E., Kelha, V., McComas, D. J., Sittler, E. C., Svenes, K. R., Szegö, K., Tanskanen, P., Ahola, K., Anderson, D., Bakshi, S., Baragiola, R. A., Barraclough, B. L., Black, R. K., Bolton, S., Booker, T., Bowman, R., Casey, P., Crary, F. J., Delapp, D., Dirks, G., Eaker, N., Funsten, H., Furman, J. D., Gosling, J. T., Hannula, H., Holmlund, C., Huomo, H., Illiano, J. M., Jensen, P., Johnson, M. A., Linder, D. R., Luntama, T., Maurice, S., McCabe, K. P., Mursula, K., Narheim, B. T., Nordholt, J. E., Preece, A., Rudzki, J., Ruitberg, A., Smith, K., Szalai, S., Thomsen, M. F., Viherkanto, K., Vilppola, J., Vollmer, T., Wahl, T. E., Wüest, M., Ylikorpi, T., and Zinsmeyer, C.: Cassini Plasma Spectrometer investigation, Space. Sci. Rev., 114, 1-112, 2004. 